\title{
How does star formation proceed in the circumnuclear starburst ring of NGC $6951 ?^{\star, \star \star}$
}

\author{
T. P. R. van der Laan ${ }^{1}$, E. Schinnerer ${ }^{1}$, E. Emsellem ${ }^{2}$, L. K. Hunt ${ }^{3}$, R. M. McDermid ${ }^{4}$, and G. Liu ${ }^{5}$ \\ 1 Max-Planck-Institut für Astronomie, Königstuhl 17, 69117 Heidelberg, Germany \\ e-mail: vanderlaan@iram. fr \\ 2 European Southern Observatory, Karl-Schwarzschild-Str 2, 85748 Garching, Germany \\ 3 INAF - Osservatorio Astrofisico di Arcetri, Largo E. Fermi 5, 50125 Firenze, Italy \\ ${ }^{4}$ Gemini Observatory, Northern Operations center, 670 N. A'ohoku Place, Hilo, HI 96720, USA \\ 5 Center for Astrophysical Sciences, Johns Hopkins University, 3400 North Charles Street, Baltimore, MD 21218, USA
}

Received 24 August 2012 / Accepted 8 January 2013

\begin{abstract}
Gas inflowing along stellar bars is often stalled at the location of circumnuclear rings, which form an effective reservoir for massive star formation and thus shape the central regions of galaxies. However, how exactly star formation proceeds within these circumnuclear starburst rings is the subject of debate. Two main scenarios for this process have been put forward. In the first, the onset of star formation is regulated by the total amount of gas present in the ring with star forming starting, once a mass threshold has been reached, in "random" positions within the ring like "popcorn". In the second, star formation primarily takes place near the locations where the gas enters the ring. This scenario has been dubbed "pearls-on-a-string". Here we combine new optical IFU data covering the full stellar bar with existing multiwavelength data to study the $580 \mathrm{pc}$ radius circumnuclear starburst ring in detail in the nearby spiral galaxy NGC 6951. Using Hubble Space Telescope (HST) archival data together with SAURON and OASIS IFU data, we derive the ages and stellar masses of star clusters, as well as the total stellar content of the central region. Adding information on the molecular gas distribution, stellar and gaseous dynamics, and extinction, we find that the circumnuclear ring in NGC 6951 is $\sim 1-1.5$ Gyr old and has been forming stars for most of that time. We see evidence for preferred sites of star formation within the ring, consistent with the "pearls-on-a-string" scenario, when focusing on the youngest stellar populations. The ring's longevity means that this signature is washed out when older stellar populations are included in the analysis.
\end{abstract}

Key words. galaxies: individual: NGC 6951 - galaxies: ISM - galaxies: stellar content

\section{Introduction}

Secular evolution comprises all agents of change in a galaxy that are independent of the external environment of the galaxy. Secular evolution, unlike hierarchical clustering and mergers, takes place on long timescales, nearly equal to the lifetime of galaxies, and it is important for a galaxy's evolution in the current epoch. A large part of secular evolution is the result of asymmetries in the gravitational potential of a galaxy. All asymmetries assist in the inward and outward motion of gas across parts of the disk. One of the asymmetries that acts the fastest and most severely is a large scale bar: an elongated concentration of stars and/or gas centered on the nucleus, usually several $\mathrm{kpc}$ in size. Depending on the strength of the bar, the sound speed in the gas, and the central mass concentration, a circumnuclear ring will form near the inner Lindblad resonance at a radius defined by the bar (e.g. Athanassoula 1992a; Regan \& Teuben 2003; Mazzuca et al. 2008; Kim et al. 2012).

A ring directly hinders further inflow towards the nucleus, i.e. nuclear feeding (e.g. Maciejewski 2004; García-Burillo et al. 2005). A circumnuclear ring is in effect the gas reservoir for the

\footnotetext{
* Tables 4 and 5 are available in electronic form at http://www . aanda.org

$\star \star$ OASIS maps and SAURON cube are available at the CDS via anonymous ftp to cdsarc.u-strasbg. fr (130.79.128.5) or via http://cdsarc.u-strasbg.fr/viz-bin/qcat?]/A+A/551/A81
}

gas moving inward. Determination of the lifetimes of circumnuclear rings is therefore paramount for understanding the influence they have on the evolution of their host galaxies. The high gas densities reached in circumnuclear rings naturally lead to star formation. The ages of the stellar population(s) in circumnuclear rings may be good indicators of the overall age of the ring, and thus of the stellar bar itself. A caveat naturally follows; how does star formation proceed in circumnuclear rings? Is there a timescale that can be related to the onset of star formation in the ring (and that should be added to determine the ring's age)?

Much theoretical and modeling work has been done on the formation of circumnuclear rings (e.g. Athanassoula 1992b; Englmaier \& Shlosman 2000; Regan \& Teuben 2003; Maciejewski 2004). Circumnuclear gas rings are closely related to the more generic nuclear spirals, because asymmetries in the gravitational potential induce a wave perturbation in the gas. All gas has angular momentum, and it needs to lose this momentum before it can move towards the center. This loss happens in the dust lanes and nuclear spirals. They are the locations where gas is shocked and loses angular momentum, allowing gas to migrate to smaller orbits.

As has been shown by Athanassoula (1992a), Regan \& Teuben (2003), and others, the existence of a circumnuclear ring inside a large-scale bar depends, among other factors, on the orbit families that make up the circumnuclear region. The $x_{1}$ orbits are elongated along the asymmetric potential and are the main 
orbits that make up the large scale bar. The $x_{2}$ orbits on the other hand, are oriented perpendicular to the bar. Gas-orbits slowly turn from one to the other in a bar potential. That $x_{2}$ orbits are again symmetric to the large-scale stellar bar potential is important, because there should be no net gravitational torques over the orbits (i.e. no further shocks in the gas). These $x_{2}$ orbits are therefore able to serve as repositories for gas. If there is a significant fraction of $x_{2}$ orbits present in the circumnuclear region, gas can settle on them and build up a circumnuclear gas ring.

The connection between dust lanes, nuclear spirals, and circumnuclear rings is very clear observationally. The dust/gas lanes of the bar do not extend to the nucleus in the case of a ring and actually end at the ring radius. Two points can often be seen in the gaseous ring, offset by $180^{\circ}$, where the lanes connect to the ring. They are called "contact points", following Böker et al. (2008). After being shocked and having lost most angular momentum, infalling gas then enters the circumnuclear ring at the contact points and will stay on the $x_{2}$ orbits. The immediate result of this situation is that the gas density in the ring will increase with continued inflow, with star formation as the natural consequence.

The most straightforward star formation model for circumnuclear rings has been proposed by Elmegreen (1994). The gas density will build up until it reaches a critical density for star formation. The entire ring will become unstable and star formation will proceed throughout the ring. The time scale of the onset of star formation is then directly related to the inflow rate onto the ring. This scenario is called "popcorn" by Böker et al. (2008), who themselves propose another scenario.

This second scenario proposes an azimuthal age gradient for the recently formed stars, starting at the contact points. Since gas enters the ring at two distinct positions, it stands to reason that the gas density in the ring is higher at the contact points (termed "overdensity regions", or "ODR" by the authors) than elsewhere in the ring. The critical density for star formation will be reached first at (or close to) those positions. By overall rotation of the system, these newly formed star clusters will be azimuthally distributed in the ring, while star formation continues at the contact points, like "pearls-on-a-string".

NGC 6951 is a good candidate for investigating which of these two scenarios holds true and for estimating the lifetime of the circumnuclear ring. NGC 6951 is a nearby $(24.1 \mathrm{Mpc}$, Tully 1988) SAB(rs)bc galaxy. It has a circumnuclear ring at a radius of $5^{\prime \prime}$ or $580 \mathrm{pc}$. It has a low inclination $\left(46.2^{\circ}\right.$, Haan et al. 2009), which provides a clear view of the entire ring. Its molecular gas dynamics in and around the ring have already been studied in detail by some of the authors (Van der Laan et al. 2011), as well as by others (Kohno et al. 1999; Krips et al. 2007; Storchi-Bergmann et al. 2007). The gravitational torques acting on the cold molecular gas have also been analyzed (Haan et al. 2009; Van der Laan et al. 2011). Mazzuca et al. (2008) have investigated the $\mathrm{H} \alpha$ regions in the ring and found young stellar ages $(<10 \mathrm{Myr})$ in the northern segment of the ring and older ages ( $\sim 10$ Myr for an instantaneous, or $\sim 100$ Myr for a continuous star formation scenario) in the rest of the circumnuclear ring. No other determination of the ages of the stellar populations of the ring has been published to date.

New observations and archival data retrieved for this study will be presented in Sect. 2. In Sect. 3 the known relevant properties of the ring are summarized. In Sect. 4 the extinction in the ring is derived, before detailing the detection of star clusters in Sect. 5. Section 6 contains the determination of the ages of the star clusters and determination of the ages of the overall stellar content based on SAURON data. A discussion with respect to

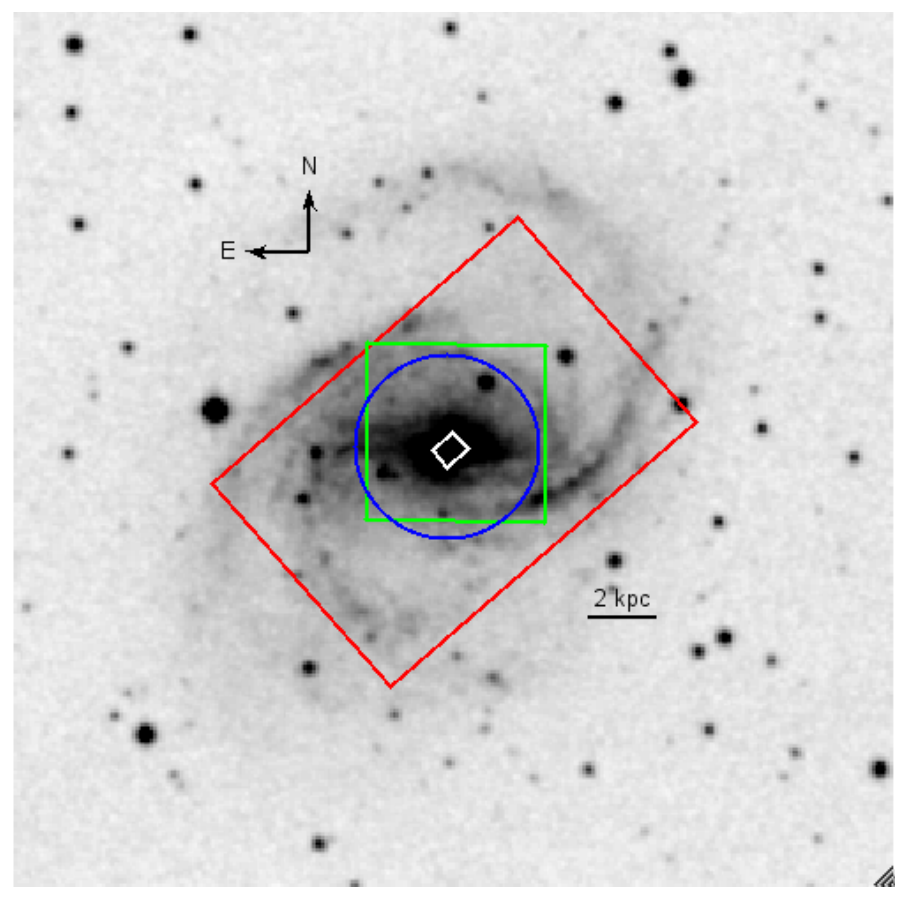

Fig. 1. Overlay of the field-of-view of various datasets presented in this paper. From small to large: OASIS (white), HST (green), PdBI (blue), SAURON (red). The underlying ( $B$ band) image was extracted from DSS.

star formation scenarios and ring lifetime is given in Sect. 7. A summary follows in Sect. 8.

\section{Observations and archival data}

\subsection{OASIS data}

The OASIS Integral-Field Unit (McDermid et al. 2004) is installed at the William Herschel Telescope (WHT) in La Palma (Canary Islands). Two individual OASIS configurations were used, namely the MR 516 and MR 661, which cover the spectral ranges around the $\mathrm{H} \beta, \mathrm{Mg}$ lines ([4760-5560 $\mathrm{A}])$ and around $\mathrm{H} \alpha$, [NII], [SII] lines ([6210-7010 ̊]), respectively, at a resolution of $R \sim 1500$, and a sampling of about $2.15 \AA$ per pixel (see the OASIS/WHT page for further details). For both configurations, the $22 \mathrm{~mm}$ enlarger was used, providing a field-of-view (FoV) of approximately 10 .' $3 \times 7$ '. 4 (see Fig. 1 ) with original spaxels of $0^{\prime \prime} 26$.

NGC 6951 was targeted during a run in July 2006, and we obtained five and four $30 \mathrm{~min}$ exposures with the MR 516 and MR 661 configurations, respectively, centered on the galaxy nucleus. Slight dithering between consecutive exposures was applied to allow for a better rejection of bad pixels or faulty spectra. Details are provided in Table 1.

All the OASIS data were reduced using the XOasis data reduction software ${ }^{1}$, developed by CRAL (Arlette Pécontal and coll.) and adapted to the above-mentioned setups. This requires the creation of a spectral mask, following a detailed model of the optical path for the instrument, which allows accurate optimal extraction of the signal from the raw exposures. The detailed reduction pipeline includes CCD corrections (bias, fringing, removal of the overscans), wavelength calibration, flatfielding (using both lamp calibrations and sky exposures), and flux calibration. Each individual exposure is then truncated to

http://www-obs.univ-lyon1.fr/labo/oasis/download/ 
Table 1. Characteristics of the SAURON and OASIS datasets.

\begin{tabular}{lccccccc}
\hline \hline IFU & Mode & \# Expo & Exp. time & Spectral sampling & Spatial sampling & Expo FoV & Total FoV \\
\hline OASIS & MR 516 & 5 & $30 \mathrm{~min}$ & $2.15 \AA$ & $0.2^{\prime \prime}$ & $10.3^{\prime \prime} \times 7.4^{\prime \prime}$ & \\
OASIS & MR 661 & 4 & $30 \mathrm{~min}$ & $2.14 \AA$ & $0.2^{\prime \prime}$ & $10.3^{\prime \prime} \times 7.4^{\prime \prime}$ & \\
SAURON & LR & 26 & $30 \mathrm{~min}$ & $1.15 \AA$ & $0.8^{\prime \prime}$ & $42^{\prime \prime} \times 33^{\prime \prime}$ & $135^{\prime \prime} \times 90^{\prime \prime}$ \\
\hline
\end{tabular}

a common wavelength domain (from $4770 \AA$ to $5530 \AA$ for MR 516, and from $6620 \AA$ to $6990 \AA$ for MR 661). We then reconstruct images by fully integrating over the spectral direction, and recenter each individual data cube so that the center of NGC 6951 corresponds to $(0,0)$. The individual exposures are then merged, taking the effect of differential refraction into account, by re-projecting the cubes onto a common spatial grid centered at $(0,0)$ with spaxels of $0.2 \times 0.2$. The final merged cubes have a spectral sampling of $2.15 \AA$ for the MR 516 configuration, and $2.14 \AA$ for the MR 661 .

\subsection{SAURON data}

NGC 6951 was observed with SAURON at the WHT (Bacon et al. 2001) during a run in August 2003. Individual exposures cover a field of view of about $41^{\prime \prime} \times 33^{\prime \prime}$, with spaxels of $0^{\prime} .94$, a spectral resolution of $4.2 \AA$ and spectral sampling of $1.15 \AA$ over the range $4780 \AA$ to $5350 \AA$. The spectral coverage of the SAURON datacubes overlaps with the OASIS MR 516, though it is significantly shorter in wavelength coverage. The coarser spatial sampling allows for a much larger spatial coverage of the disk of NGC 6951 than with OASIS (see also Table 1). We obtained a total of twenty-six 30 min exposures, the goal being to cover as much of the stellar bar as possible. The total time spent on each pointing was adopted according to the average surface brightness, and included some slight dithering for each pointing to prevent systematics. The final area covered by the complete mosaic is $135^{\prime \prime} \times 90^{\prime \prime}$ (see Fig. 1). The data from the single, central exposure has been presented previously by Dumas et al. (2007).

The data reduction of all SAURON exposures was performed using a pipeline based on XSauron that is similar to the XOasis version, but adapted to the different optics and setup. The reduction steps are thus very similar to the ones described in the previous section for OASIS. The biggest differences lie in the characteristics of the spectral mask, and the existence, for SAURON, of about 150 dedicated sky lenses that allow for an accurate sky subtraction for each exposure.

The fully calibrated individual exposures were then recentered (using a direct image of the galaxy as a reference) and merged, by projecting each data cube on a common grid with $0 .{ }^{\prime} 8 \times 0 .{ }^{\prime} 8$ spaxels. To increase the signal-to-noise ratio $(\mathrm{S} / \mathrm{N})$, the spaxels were Vonoroi binned to an $\mathrm{S} / \mathrm{N}$ of 40 .

\subsection{Spectral fitting of the IFU datasets}

We applied standard routines to extract the kinematics: the stellar kinematics (velocity, velocity dispersion and Gauss-Hermite $h_{3}$ and $h_{4}$ values) with pPXF (Cappellari \& Emsellem 2004; Emsellem et al. 2004) using a set of stellar templates extracted from the MILES library ${ }^{2}$ (Sánchez-Blázquez et al. 2006; Falcón-Barroso et al. 2011) and the IDL implementation available from Michele Cappellari's web page $^{3}$, and the

\footnotetext{
2 http://miles.iac.es

3 http://www-astro.physics.ox.ac.uk/ mxc/idl/
}

gas kinematics via a version of GANDALF (kindly adapted for our purpose by Marc Sarzi, Sarzi et al. 2006). Fitted emission lines include $\mathrm{H} \beta$, the doublets [OIII] $(\lambda(4959,5007) \AA)$, [NI] $(\lambda(5200,5202) \AA)$, in both SAURON, and OASIS MR 516, and [OI] $(\lambda(6300,6364) \AA), \mathrm{H} \alpha(\lambda 6562.8 \AA)$, and the doublets [NII] $(\lambda(6548,6583) \AA)$ and [SII] $(\lambda(6717,6731) \AA)$ for the OASIS MR 661 data cube. The kinematics of the emission lines was fixed to the [OIII] doublet (SAURON), and [OI], [NII], and $[\mathrm{SII}]$ kinematics were fixed together in OASIS MR 661. The $\mathrm{H} \alpha$ emission was strong enough to be fit independently. We emphasize that $\mathrm{H} \beta$ and $\mathrm{H} \alpha$ are present both in emission and absorption; however, the combined use of pPXF and GANDALF does take this into account.

The resulting emission line maps from the spectral fitting for OASIS are shown in Fig. 2. It can be seen that the OASIS FoV just covers the circumnuclear ring. The $\mathrm{H} \alpha$ and $\mathrm{H} \beta$ emission is mostly concentrated in the ring, while the [OI] and [OIII] emission is limited to the nucleus. The [NII] and [SII] emitting gas is present in both the nucleus and ring. There are bright hot spots in the ring that are seen in $\mathrm{H} \alpha, \mathrm{H} \beta$, [NII] and [SII]. The velocity field and dispersion are only shown for $\mathrm{H} \alpha$, but are very similar for the other emission lines. The increase in the velocity dispersion in the western half of the ring was also seen in the SAURON observations presented by Dumas et al. (2007) and coincides with the lowest intensities of $\mathrm{H} \alpha$ and $\mathrm{H} \beta$ line emission in the ring.

The spectral fitting results from the SAURON mosaic are presented in Fig. 3. In the top lefthand panel of Fig. 3 we show the total intensity over the FoV of the mosaic. The peak intensity of the central region has been saturated to show the details of the entire stellar bar region. The stellar bar is oriented approximately east-west on the sky. The few high-intensity peaks are foreground stars. The stellar velocity field and dispersion are also shown in the top row.

The $\mathrm{H} \beta$, [NI], and [OIII] emission line maps are presented in the bottom row of Fig. 3. It is clear that their emission is concentrated in the circumnuclear region. In the case of $\mathrm{H} \beta$, a keen eye can make out the ring shape of the emission in this figure (exactly as was seen in the OASIS map).

\subsection{HST images}

We retrieved F547M, F606W, F658N, F814W, and F330W images of NGC 6951 from the MAST HST archive. We extracted them from the archive calibrated with the "best" reference files. We also utilize NICMOS F110W and F160W images from the HST archive already used in previous work (Van der Laan et al. 2011, original: Hunt \& Malkan 2004). Finally, we obtained a $\mathrm{Pa} \alpha$ image, which was observed during the same campaign and reduced using the same approach as for NGC 1097 in Hsieh et al. (2011). The full sample and detailed data reduction for the $\mathrm{Pa} \alpha$ image are presented in Liu et al. (in prep.). A list of all images used is given in Table 2.

The $\mathrm{H} \alpha$ image was carefully continuum subtracted with the F606W image, as was the $\mathrm{Pa} \alpha(\mathrm{F} 190 \mathrm{~N})$ image using the 

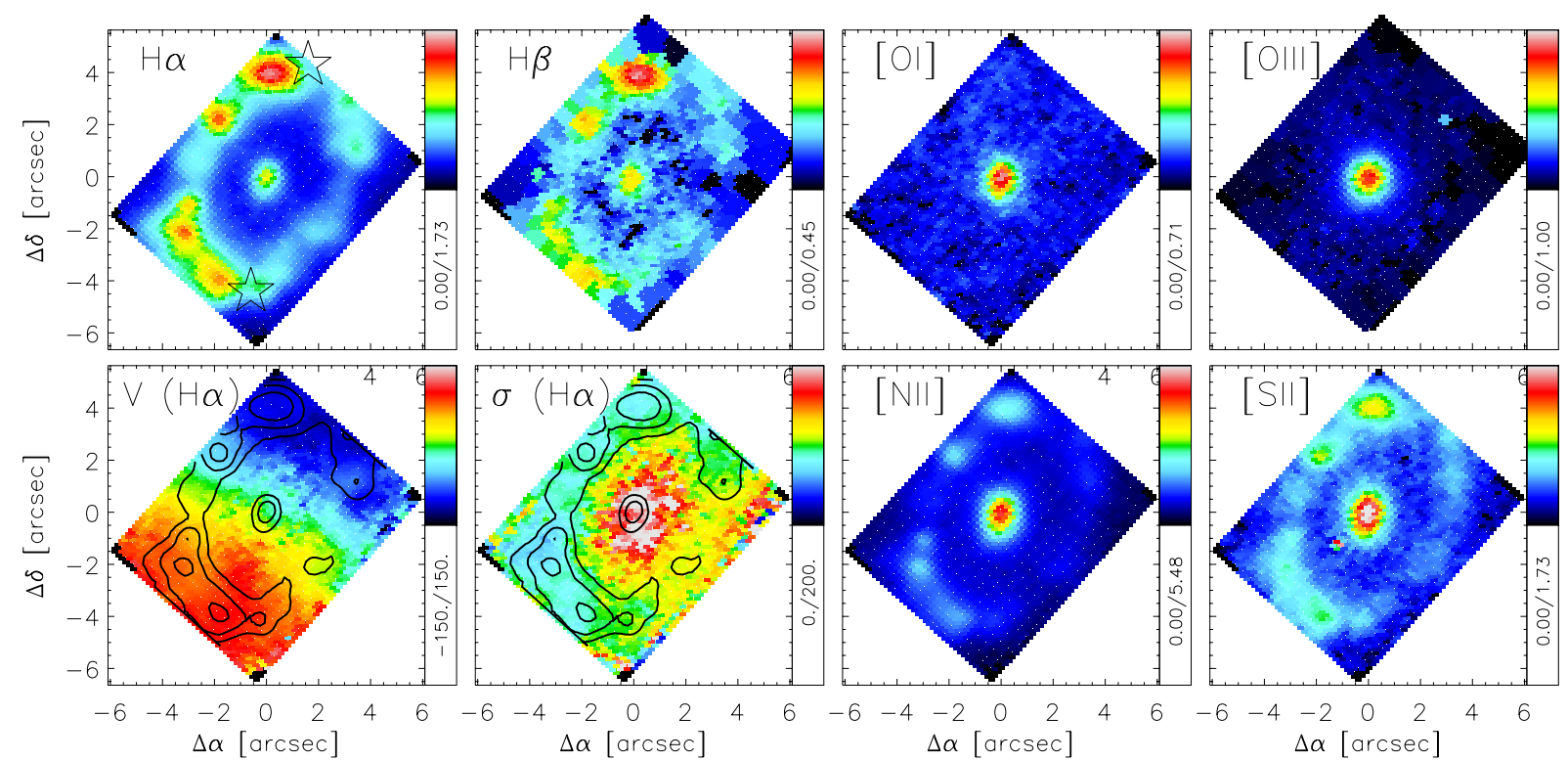

Fig. 2. Top panels and bottom right: OASIS intensity maps of the emission lines $\mathrm{H} \alpha, \mathrm{H} \beta$, [OI], [OIII], [NII] and [SII]. The color scale in each panel indicates the square of the intensity, the range of which is given below the color bar. This scaling was chosen to emphasize faint structures in the maps. The "contact points", discussed in the text, are indicated with a star in the H $\alpha$ panel. Bottom left panels: the velocity and velocity dispersion map of $\mathrm{H} \alpha$. The flux contours of the integrated $\mathrm{H} \alpha$ emission are overplotted for emphasis. The velocity range in the bottom left panel is $[-150,150] \mathrm{km} \mathrm{s}^{-1}$, with an assumed systemic velocity of NGC 6951 of $1425 \mathrm{~km} \mathrm{~s}^{-1}$. The velocity dispersion range in the adjacent panel is $[0,200] \mathrm{km} \mathrm{s}^{-1}$. The orientation of all maps is north up, east left. All positions are relative to the nucleus.
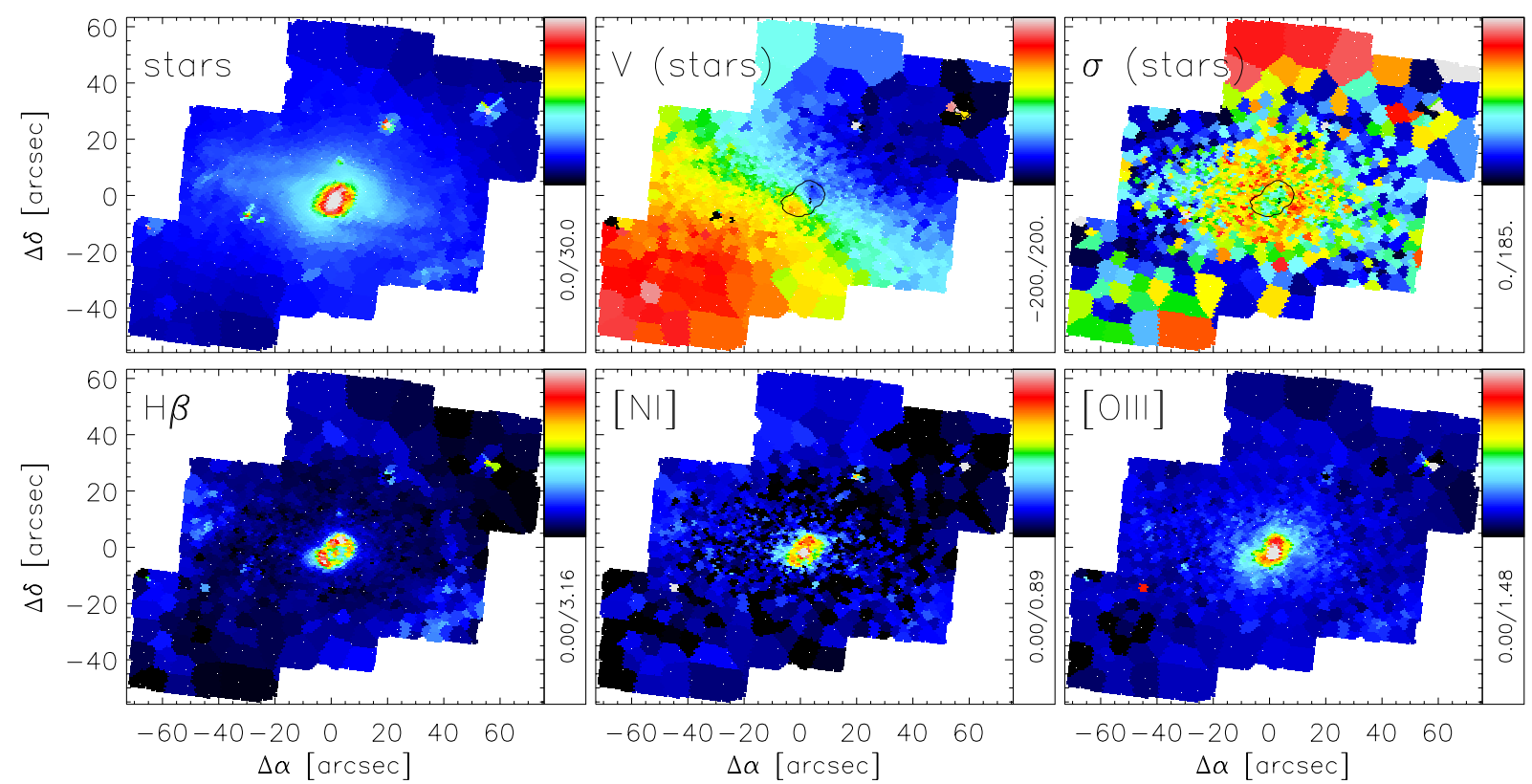

Fig. 3. SAURON intensity maps of the stellar light (top left), H $\beta$ (bottom left), [NI] (bottom middle), and [OIII] (bottom right) emission lines, as well as the stellar velocity field (top middle) and velocity dispersion map (top right). The color scale in each panel indicates the square of the intensity, or the velocity, the range of which is given below the color bar. The orientation of the maps is north up, east left. All positions are relative to the nucleus. To guide the eye, the contour corresponding to the central $\mathrm{H} \beta$ emission has been overplotted onto the velocity and velocity dispersion plots. The velocity range in the top-middle panel is $[-200,200] \mathrm{km} \mathrm{s}^{-1}$, the assumed systemic velocity of NGC 6951 is $1425 \mathrm{~km} \mathrm{~s}^{-1}$. The velocity dispersion range in the top-right panel is $[0,185] \mathrm{km} \mathrm{s}^{-1}$.

F187N image. The F658N filter passband includes emission from the [NII] double line emission. From spectra presented in Storchi-Bergmann et al. (2007), the ratio [NII]6583 $\AA / \mathrm{H} \alpha$ is known to be $\sim 0.3$ throughout the ring, implying a $37 \%$ contribution to the observed flux from [NII] $6548 \AA$ and [NII] $6583 \AA$ in the HST F658N filter for a $V_{\text {sys }}$ of $1425 \mathrm{~km} \mathrm{~s}^{-1}$, for which we correct.

\section{NGC 6951 's circumnuclear ring so far...}

Before starting the analysis of the stellar properties of NGC 6951's circumnuclear ring, we present a brief summary of the gaseous properties of the circumnuclear region that are known to date. For clarity later on, all numbers pertinent to the discussion later are also listed in Table 3. The large scale stellar 
Table 2. HST archival data.

\begin{tabular}{lcccccc}
\hline \hline Filter & Program ID & Exposure [s] & Obs. date & PHOTFLAM & Instrument & Scale ["/pixel] \\
\hline$F 187 N^{*}$ & 11080 & 1151.767 & $13-02-2007$ & $3.6837500 \times 10^{-18}$ & NICMOS NIC3 & 0.10 \\
$F 160 W$ & 7331 & 255.923 & $16-12-1997$ & $2.3600094 \times 10^{-19}$ & NICMOS NIC2 & 0.076 \\
$F 110 W$ & 7331 & 255.923 & $16-12-1997$ & $4.3320170 \times 10^{-19}$ & NICMOS NIC2 & 0.076 \\
$F 547 M$ & 5419 & 300 & $16-07-1994$ & $7.691168 \times 10^{-18}$ & WFPC2/PC & 0.046 \\
$F 606 W$ & 8597 & 160 & $18-11-2000$ & $1.899711 \times 10^{-18}$ & WFPC2/PC & 0.046 \\
$F 658 N$ & 9788 & 700 & $24-10-2003$ & $1.9597158 \times 10^{-18}$ & ACS WFC1 & 0.05 \\
$F 814 W$ & 9788 & 120 & $24-10-2003$ & $6.9255574 \times 10^{-20}$ & ACS WFC1 & 0.05 \\
$F 330 W$ & 9379 & 1200 & $17-08-2002$ & $2.2688470 \times 10^{-18}$ & ACS HRC-FIX & 0.025 \\
\hline
\end{tabular}

Notes. List of the observations extracted from the HST archive. ${ }^{(*)}$ The Pa $\alpha$ image has been corrected for continuum flux with an F190N-filter image taken at the same time.

Table 3. Properties of NGC 6951.

\begin{tabular}{lcc}
\hline \hline Parameter & Value & Reference \\
\hline Type & SAB(rs)bc & $(1)$ \\
Nuclear activity & LINER/Seyfert 2 & $(2)$ \\
\multicolumn{1}{c}{ Dynamical center (Locus radio continuum) } & \\
RA (J2000) & $20^{\mathrm{h}} 37^{\mathrm{m}} 14.123^{\mathrm{s}}$ & $(3,4)$ \\
Dec (J2000) & $66^{\circ} 06^{\prime} 20.09^{\prime \prime}$ & $(3,4)$ \\
Inclination angle & $46.2^{\circ}$ & $(5)$ \\
Position angle & $138^{\circ}$ & $(5)$ \\
Adopted distance & $24.1 \mathrm{Mpc}$ & $(6)$ \\
Stellar bar PA & $84^{\circ}$ & $(7)$ \\
Mass mol. gas $(r<3 \mathrm{kpc})$ & $1.6 \times 10^{9} M_{\odot}$ & $(8)$ \\
Mol. gas inflow rate onto ring & $2.0 M_{\odot} / \mathrm{yr}$ & $(8)$ \\
Large scale bar pattern speed & $28.9 \pm 1.2 \mathrm{~km} \mathrm{~s} \mathrm{~s}^{-1} / \mathrm{kpc}$ & $(9)$ \\
$V_{\text {sys }}$ & $1425 \mathrm{~km} \mathrm{~s} \mathrm{~s}^{-1}$ & $(5)$ \\
Period pattern speed & $210 \mathrm{Myr}$ & $(9)$ \\
1 rotation for a ring orbit & $24 \mathrm{Myr}$ & $(9)$ \\
\hline
\end{tabular}

References. (1) de Vaucouleurs et al. (1991); (2) Pérez et al. (2000); (3) LEDA, Krips et al. (2007); (4) Saikia et al. (2002); (5) Haan et al. (2009); (6) Tully (1988); (7) Mulchaey \& Regan (1997); (8) Van der Laan et al. (2011); (9) this work.

bar in NGC 6951 has a $P A$ of $84^{\circ}$ on the sky (Mulchaey \& Regan 1997). Its semi-major axis is $3.0 \mathrm{kpc}$. The dust lanes of the bar are thus oriented almost horizontally on the sky. The contact points in the ring are in the north and south of the ring (see also Figs. 2 and 4$)$.

The ${ }^{12} \mathrm{CO}(1-0)$ and ${ }^{12} \mathrm{CO}(2-1)$ observations, taken with the IRAM PdBI and $30 \mathrm{~m}$ telescope, of the circumnuclear region of NGC 6951 are presented in Van der Laan et al. (2011). The resolution of the $\mathrm{CO}(1-0)$ observations is $3.11^{\prime \prime}$ by $2.59^{\prime \prime}$, the $\mathrm{CO}(2-1)$ observations are $1.72^{\prime \prime}$ by $1.56^{\prime \prime}$. The FoV of the $\mathrm{CO}(1-0)$ observations is shown in Fig. 1, the FoV of the $\mathrm{CO}(2-1)$ data is about half that size. Both completely cover the ring. Their rms noise corresponds to $M_{\mathrm{H}_{2}} \sim 10^{5} M_{\odot} /$ beam. We refer to Van der Laan et al. (2011) for further details on the data reduction. A total $\mathrm{H}_{2}$ molecular gas mass of $2.2 \times$ $10^{9} M_{\odot}$ was derived from the $\mathrm{CO}(1-0)$ observations, assuming a Galactic conversion factor and corrected for helium abundance. An $\mathrm{HCN}(1-0)$ intensity map obtained with PdBI was presented by Krips et al. (2007). A map with slightly different $u v$-weighting from that dataset was kindly provided by Krips (priv. comm.), with a beam size of $1.89^{\prime \prime}$ by $1.83^{\prime \prime}\left(\mathrm{PA} 78.7^{\circ}\right.$ ).

The key points for the molecular gas morphology are prominent gas spiral arms, which are co-spatial with the dust lanes along the large-scale stellar bar and which end at the radius of

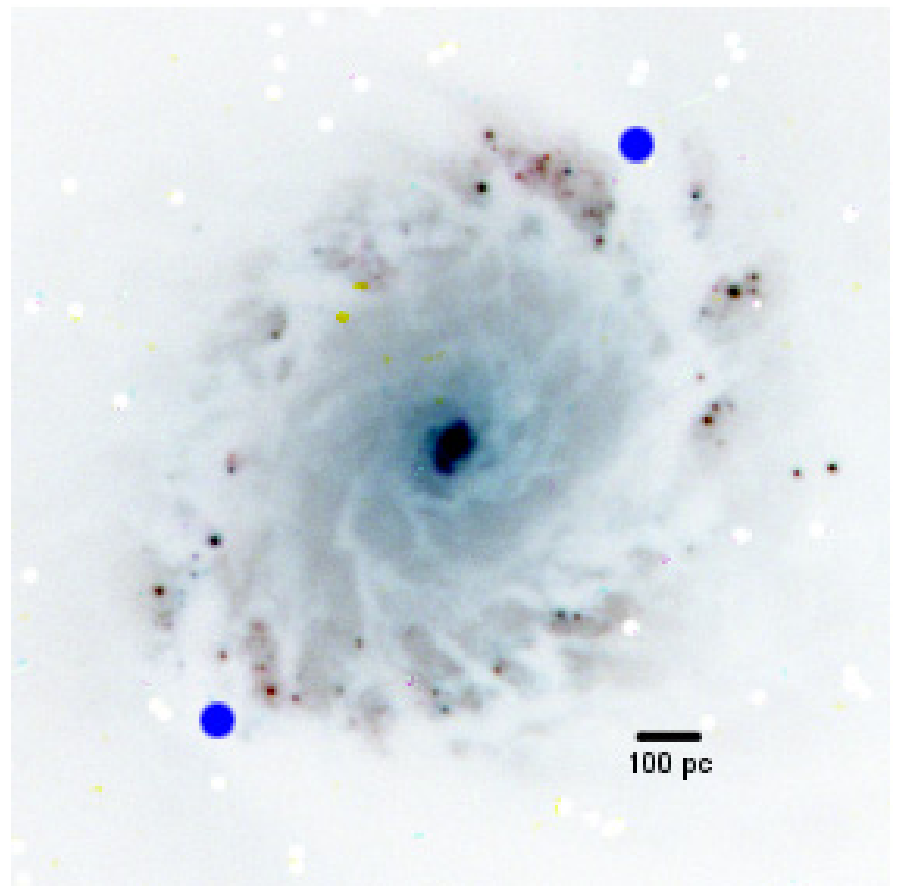

Fig. 4. False color image of the circumnuclear ring in NGC 6951, based on the F814W (red), F606W (green), and F547M (blue) broad band filters. The image $\left(\sim 14^{\prime \prime} \times \sim 14^{\prime \prime}\right)$ is oriented with north pointing up and east to the left. The center has been saturated for better visibility of the ring, and bad pixels have been removed. The "contact points" of the ring are indicated with blue circles.

the circumnuclear ring. The $\mathrm{CO}(2-1)$ observations, which have a higher spatial resolution, show that the spiral arms continue into a ring. The $\mathrm{HCN}(1-0)$ traces higher density molecular gas than $\mathrm{CO}$, and the $\mathrm{HCN}$ intensity distribution shows a similar morphology to the $\mathrm{CO}(2-1)$ molecular gas. However, the intensity maxima are at slightly different positions in the ring. From modeling the orbits in the circumnuclear region, Van der Laan et al. (2011) derived that the inflow rate of gas onto the ring is $\sim 2.0 M_{\odot}$ per year.

The HI distribution, presented in Haan et al. (2009), shows that the circumnuclear region of NGC 6951 is HI-deficient. The neutral ISM in the circumnuclear region is thus dominated by molecular gas. Given an estimate for the dynamical mass at $1 \mathrm{kpc},\left(M_{\mathrm{dyn}}=\frac{r v^{2}}{G} \approx 8 \times 10^{9} M_{\odot}\right.$, with $\left.v=200 \mathrm{~km} \mathrm{~s}^{-1}\right)$, atomic and molecular gas makes up approximately $25 \%$ of the dynamical mass within this radius. 

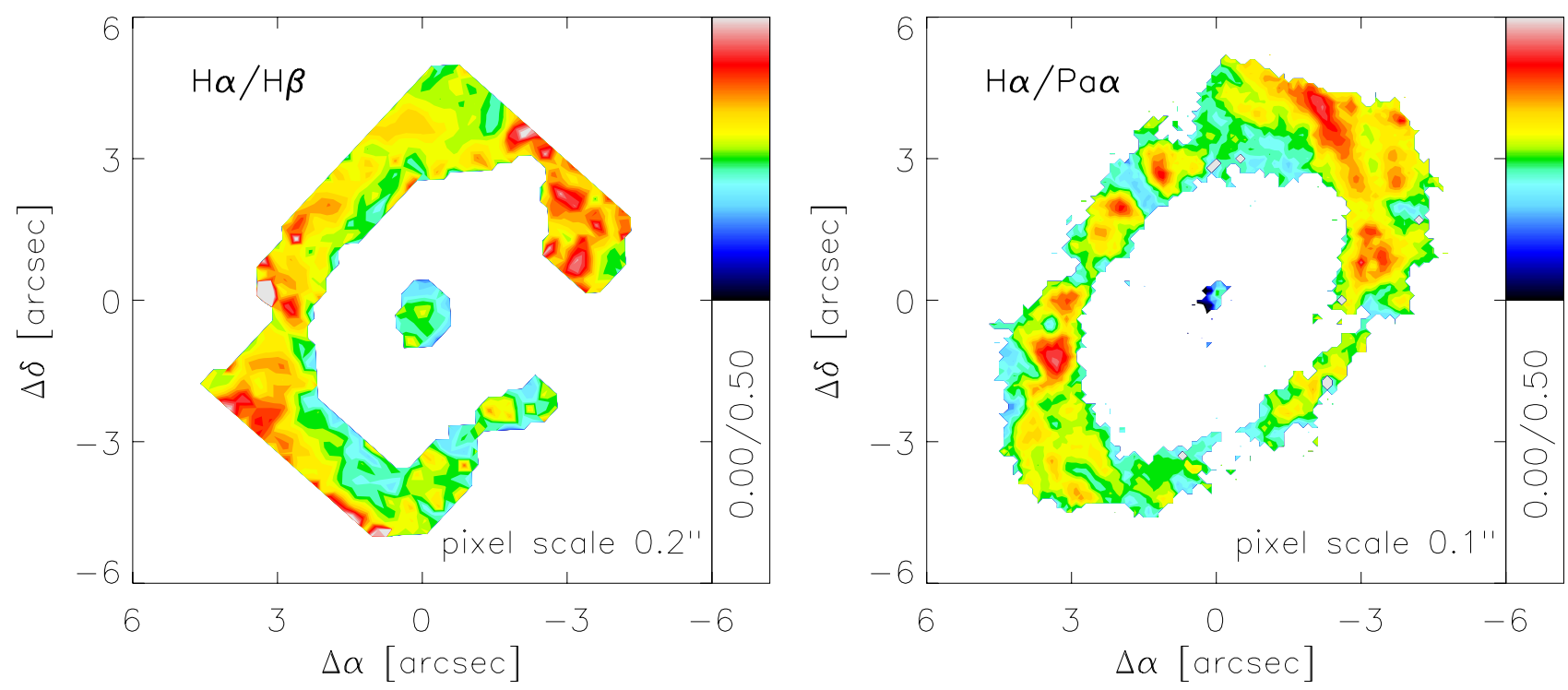

Fig. 5. Left: color excess $E(B-V)$ in the ring as measured from the $\mathrm{H}_{\alpha} / \mathrm{H}_{\beta}$ line ratio using the OASIS line maps. Right: color excess in the ring as measured from the $\mathrm{H} \alpha / \mathrm{Pa} \alpha$ line ratio using HST images. The pixel scales of each map are given in the lower right corner of each panel.

Two determinations of the large-scale bar pattern speed of the stellar bar in NGC 6951 exist in the literature. Aguerri et al. (1998) obtained a value of $\Omega_{\mathrm{p}}=30.3 \mathrm{~km} \mathrm{~s}^{-1} / \mathrm{kpc}$ (when compensating for the difference in adopted distance) based on the rotation curve and location of the corotation radius and Haan et al. (2009) derived a value of $\Omega_{\mathrm{p}}=23 \mathrm{~km} \mathrm{~s}^{-1} / \mathrm{kpc}$ based on an analysis of dynamic resonances. The SAURON IFU mosaic covers most of the large-scale stellar bar of NGC 6951, and is ideally suited to measuring the pattern speed using the modelfree Tremaine-Weinberg method (Tremaine \& Weinberg 1984). Using the SAURON mosaic gives a value of $28.9 \pm 1.2 \mathrm{~km} \mathrm{~s}^{-1} / \mathrm{kpc}$, corrected for inclination $\left(46.2^{\circ}\right)$ and at a distance of $24.1 \mathrm{Mpc}$. Details will be available in a forthcoming paper.

With a pattern speed of $28.9 \mathrm{~km} \mathrm{~s}^{-1} / \mathrm{kpc}$, the pattern will take 210 Myr to complete one rotation. The gas and stars rotate with a velocity of $\sim 150 \mathrm{~km} \mathrm{~s}^{-1}$ at the ring radius. They will thus complete a rotation in the ring in $24 \mathrm{Myr}$.

\section{Extinction in the ring}

From the three hydrogen recombination lines $(\mathrm{H} \alpha, \mathrm{H} \beta$, and $\mathrm{Pa} \alpha)$ in our data sets we can compute a spatial distribution of the extinction in the ring. While determining the extinction is not directly crucial to the overarching goal in this work, it provides a consistency check on the derived color excesses of the star cluster fitting (Sect. 5). We use two different ratios to compute the extinction, the $\mathrm{H} \alpha / \mathrm{H} \beta$ ratio (as derived from our OASIS data), and the $\mathrm{H} \alpha / \mathrm{Pa} \alpha$ ratio (as derived from the HST data).

Both the $\mathrm{H} \alpha$ and $\mathrm{H} \beta$ maps are obtained during the spectral fitting of the OASIS data cubes. The fitting takes $\mathrm{H} \alpha$ and $\mathrm{H} \beta$ stellar absorption into account as explained in Sect. 2.3. In the case of case $\mathrm{B}$ recombination, the ratio $\mathrm{H} \alpha / \mathrm{H} \beta$ will be 2.86 (Osterbrock 1989). Extinction will increase the value. In individual pixels, we find ratios as high as $\sim 4$, which is equal to an extinction of $\sim 1 \mathrm{mag}$. Inside the ring, the surface brightness of especially $\mathrm{H} \beta$ is so low that no reliable $\mathrm{H} \alpha / \mathrm{H} \beta$ ratio can be computed. Where that is the case, the position has been left blank. In Fig. 5 (right) the color excess, $E(B-V)$ is plotted, based on the $\mathrm{H} \alpha / \mathrm{H} \beta$ ratio. Color excess is a wavelength independent measure of extinction related to extinction via $E(B-V)=A_{V} / R_{V}$, where $R_{V}$ gives the total-to-selective extinction, which we assume to be 4.05 (starburst value of Calzetti et al. 2000).

The FoV of OASIS only barely covers the entire ring. Nevertheless, it is possible to see some trends in the $\mathrm{H} \alpha / \mathrm{H} \beta$ map. The extinction is clearly higher near and "downstream" of the contact points between ring and bar-induced gas lanes (galaxy rotation is clockwise).

The ratio of the HST $\mathrm{H} \alpha$ and $\mathrm{Pa} \alpha$ observations provides another measure of the extinction. The advantages of these observations are the complete view of the ring and higher spatial resolution (Fig. 5, left). Without extinction the expected ratio $\mathrm{H} \alpha / \mathrm{Pa} \alpha$ is about 8.5 (Hummer \& Storey 1987, case B recombination). Inside the ring the intensity of both $\mathrm{H} \alpha$ and $\mathrm{Pa} \alpha$ is too low to obtain reliable ratios. Those positions have been left blank. Again we see the highest extinctions "downstream" of the contact points. In the western part of the ring we also find "pockets" of higher extinction that can be related to the highest intensity $\mathrm{CO}$ and $\mathrm{HCN}$ peaks. These places are heavily obscured and dense, and thus are likely the sites of future star formation. Comparison with the $\mathrm{H} \alpha$ distribution shows the brightest $\mathrm{H} \alpha$ regions to be anti-correlated with extinction.

The $\mathrm{H} \alpha / \mathrm{H} \beta$ based color-excess map (OASIS) and the $\mathrm{H} \alpha / \mathrm{Pa} \alpha$ based color-excess map (HST) show similar distributions, and have similar values in the range [0.2-0.5] mag.

\section{Star clusters}

Star clusters are a prime way to study the ages of stellar populations, because all the stars within a cluster are coeval and clusters are bright and compact, thus easily observable even over long distances. At the distance of NGC 6951 star clusters are point sources and can easily be separated from the background light in the galaxy.

\subsection{Identification}

Two independent routines were used for the detection and photometry of the star clusters in the ring of NGC 6951. These 
routines are the DAOPHOT package (Stetson 1987; Stetson et al. 1990; Stetson 1992) in IRAF and SExtractor (Bertin \& Arnouts 1996). With this approach, the strengths of both methods can be exploited to obtain the most complete star cluster candidate list.

For the detection of point sources, a composite optical image was created out of four individual optical bands; F547M, F606W, F814W, and F330W. First, the F606W, F814W, and F330W images were aligned with the F547M image using standard procedures in IRAF. For the F814W and F330W bands, the images were resampled to the F547M pixel size while conserving the flux. The four resulting images were then normalized and added together.

Applying DAOFIND, 33 star cluster candidates were detected in the composite optical image (within $r \sim 20^{\prime \prime}$ ). The point source detection in the NIR images was done separately from the composite optical image, since the NIR images have a lower angular resolution. The F110W image was used to detect the NIR star clusters, and the resulting positions were applied to the F160W image. Most of the NIR detections have a counterpart in the composite optical image, but several sources were detected without one. This leads to a total of 42 detected star cluster candidates using the DAOPHOT method.

Next, SExtractor was run. The strength of SExtractor is its ability to "de-blend" crowded regions. As can be seen from the false color image (Fig. 4), especially the northern region of the ring is very crowded. For detection and the subsequent photometry of star cluster locations, the same composite optical image from the DAOFIND analysis was used. SExtractor automatically determines apertures around the selected source. If the individual images were used independently, the selected apertures between the different wavelengths could vary, which would introduce systematic changes in our photometry. By using the composite optical image as the "detection image" this is avoided, and each source will have the same aperture at the different wavelengths.

The SExtractor output is filtered by discarding all detections flagged as "bad" by SExtractor itself (e.g. likely to be cosmic rays or one or more pixels that are saturated). The likely candidates are further constrained by location (a rectangular area around the ring of $\left.12^{\prime \prime} \times 12^{\prime \prime}\right)$ and by relative error in the measured flux $(<20 \%)$. Forty-three star cluster candidates are detected.

In total, 55 different star cluster candidates are detected (see Table 4), about half of which are detected by both DAOPHOT and SExtractor (28 out of 55 or $51 \%$ ).

\subsection{Properties}

The 55 star cluster candidates can be compared with the work by Barth et al. (1995), who used a single F547M image (the same one we extracted from the archive). All 24 sources already presented in that work are also included in our list. The additional candidates lie in the northern region and several have lower surface brightnesses. Investigation of the F330W image, which has the finest native pixel scale, shows that some of our detections, particularly those in the crowded northern region of the ring, are actually still composed of two or multiple point sources/star clusters.

SExtractor simultaneously detects the star cluster candidates and measures their flux. For the star cluster candidates identified with DAOFIND, their photometric fluxes were obtained using PHOT (also in the IRAF/DAOPHOT package). For the measurement the F330W and F814W images are used at the resolution of the F547M and F606W images. In PHOT the circular apertures for the optical images were chosen to have a radius

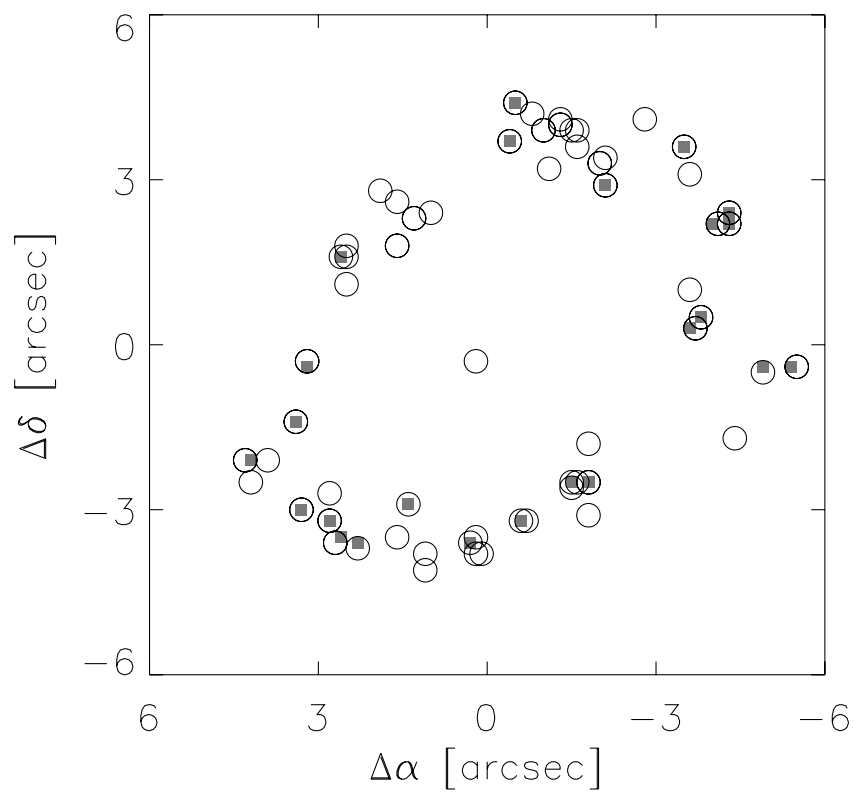

Fig. 6. Locations of the candidate star clusters as detected with IRAF/DAOPHOT and SExtractor (open circles). We have also indicated the locations of the star clusters found by Barth et al. (1995) (gray squares). The axes denote the projected distances in arcseconds from the photometric nucleus, see Table 3.

of 2 pixels $\left(0.09^{\prime \prime}\right)$. This radius contains most of the observed $\mathrm{PSF}$ ( 4 pixels $\approx F W H M \sim 20 \mathrm{pc}$ ), while minimizing the contamination from other sources (such as other close-by star clusters). The sky subtraction was based on an annulus around each source with radii between 6 and 16 pixels. Flux outliers in this annulus (e.g. other clusters or cosmic-rays, which would overestimate the skylevels) were automatically excluded from the sky estimate. For the NIR images, an photometry aperture radius of 1.2 pixels was used, which corresponds to a size equal to the optical aperture. The radii of the sky estimate annulus were again 6 and 16 pixels for the NIR images.

The TinyTim package ${ }^{4}$ was used to obtain the necessary aperture corrections for each band. The aperture corrections are for the $F 330 \mathrm{~W}$ band $1.16 \times f_{330}, F 547 M$ band $1.29 \times f_{547}, F 606 \mathrm{~W}$ band $1.31 \times f_{606}, F 658 \mathrm{~N}$ band $1.40 \times f_{658}, F 814 \mathrm{~W}$ band $1.36 \times f_{814}$, $F 110 W$ band $1.38 \times f_{110}$, and $F 160 W$ band $1.59 \times f_{160}$.

All measured photometric fluxes are converted into absolute magnitudes, using a distance modulus of 31.9 mag. Barth et al. (1995) used a distance modulus of 31.2, which corresponds to a distance of $17.4 \mathrm{Mpc}$. Assuming a distance of $24.1 \mathrm{Mpc}$ to NGC 6951, as in Van der Laan et al. (2011), increases the distance modulus to 31.9 , or correspondingly increases the magnitudes by $-0.7 \mathrm{mag}$. The absolute magnitudes are given in Table 4 .

The absolute magnitudes from both methods are in good agreement with each other and with Barth et al. (1995), with at most a 0.5 mag difference in the optical bands and $1 \mathrm{mag}$ in the NIR bands, mostly due to differences in sky subtraction. These values have not (yet) been corrected for extinction. A measure for the intrinsic extinction is derived during the star cluster fitting.

4 http://www.stsci.edu/software/tinytim/tinytim.html 

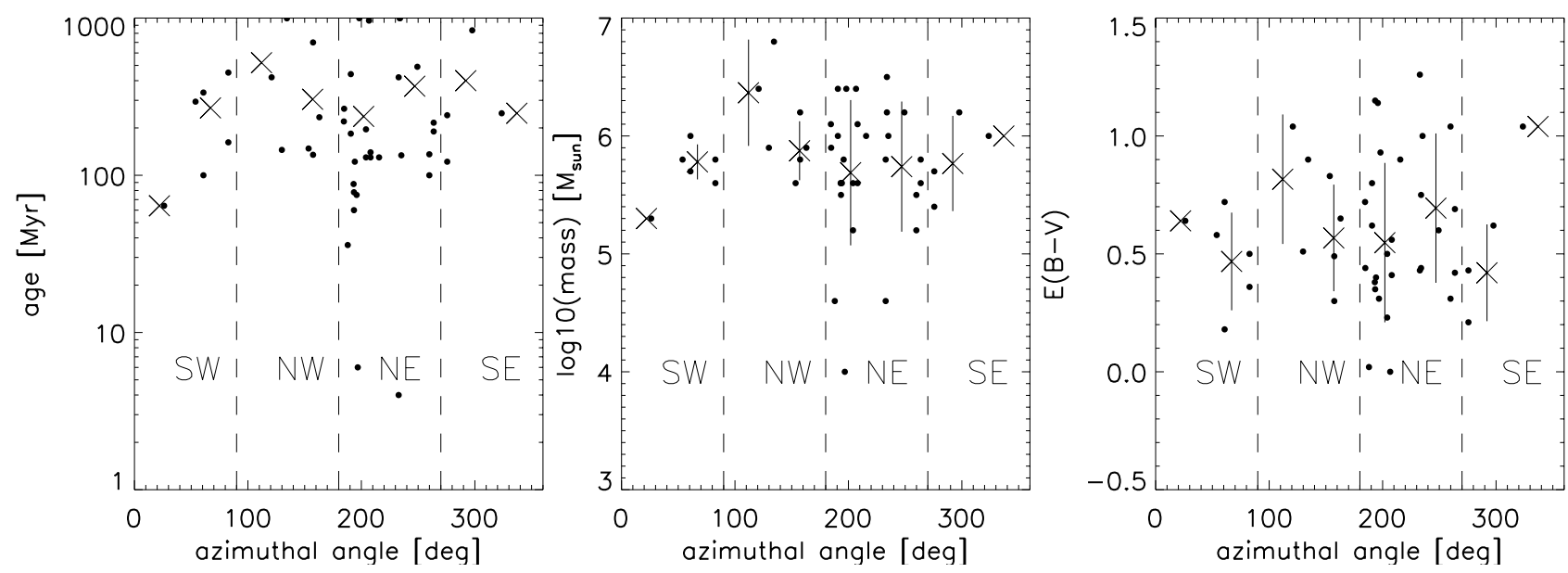

Fig. 7. Results of the $\chi^{2}$ fitting. The four segments of the ring are indicated in the plots. From left to right are shown: stellar age, mass, and color excess of the cluster. The individual results are averaged every $45^{\circ}$ (crosses), and the variance is indicated with the error bars. The angle starts at the southern point of the ring and is measured counter-clockwise.

\section{Ages of the stellar populations in the ring}

Determining the ages of the stars in the ring gives information on the age of the ring in NGC 6951 itself, since the star formation occurs in the circumnuclear ring.

\subsection{Starclusters}

The online Starburst99 (v6.0.2) code (Leitherer et al. 1999; Vázquez \& Leitherer 2005; Leitherer et al. 2010) was run to obtain model SEDs for star clusters of different ages. We assumed a fixed stellar mass of $10^{5} M_{\odot}$, an instantaneous star formation event with a Kroupa IMF, and Padova AGB solar metallicity stellar tracks. The model SEDs span the UV to the NIR in the time range of $10^{4}$ to $10^{9}$ years in steps of $2 \times 10^{6}$ years. We also include nebular emission in the model fluxes, which adds significant emission for young ages at, predominantly, the shorter wavelengths.

For comparison to our observed data points, these model spectra are weighted with a range of $E(B-V)$ values $([0,3]$ in 0.01 steps) and stellar masses $\left(\left[10^{3}, 10^{7} M_{\odot}\right]\right.$, in 0.2 dex steps). Mass scales linearly with luminosity and, as such, scales to the observed SED, independent of wavelength. A different distance modulus (31.2 instead of 31.9) would have a similar effect. To convert the color excess into an extinction curve, the Calzetti extinction law (Calzetti et al. 2000) for starburst galaxies is used. The Starburst99 spectra so obtained are convolved with the different HST filter throughput curves, and converted to magnitudes. A $\chi^{2}$-fit is applied to each star cluster set of observed magnitudes. The lowest $\chi^{2}$ value is selected as the best fit to our data (Table 5).

Table 5 shows that the best fit ages for the star clusters span young (4 Myr) to intermediate (300 Myr) ages, with several older clusters present $(\gtrsim 700 \mathrm{Myr})$. The stellar mass range of the star clusters is $\sim 10^{4.0}$ to $\sim 10^{6.8} M_{\odot}$, and the $E(B-V)$ color excess varies from 0 to $1.0 \mathrm{mag}$, with most star clusters having a color excess of $<0.6$. The results of the star cluster fitting are also shown as a function of azimuthal position in the ring in Fig. 7. The four quadrants (SW, NW, NE, and SE) should be visualized as overlaying compass points on the ring and are chosen with the contact points between gas/dust lanes of the large-scale bar and the ring in mind. These points fall just inside the SW and NE quadrants. The rotation sense of the galaxy is clockwise, which corresponds to a decreasing angle. The results are averaged every $45^{\circ}$ to detect any azimuthal trends in age, mass, or color excess.

The star clusters predominantly have intermediate age and are massive, which explains why they are still detectable. From the lefthand panel (age) in Fig. 7 it can be seen that the star cluster ages in the ring vary little, although they do drop in the SW and NE quadrant, before rising. It is not clear whether this trend could be a weak age gradient. Mazzuca et al. (2008) find a qualitatively similar age distribution, although we should point out that 100 Myr ages for the $\mathrm{H} \alpha$ emission region in that work can only be achieved with a continuous star formation history. The star clusters are assumed to have formed in a single burst. However, the $\mathrm{H} \alpha$ regions discussed in Mazzuca et al. (2008) cover a much larger area than the individual star clusters in this work. Thus, both results would agree if some younger stellar population is not or no longer concentrated in star clusters, but is present in the ring. We discuss this scenario further in the next section.

The color excess derived from the star cluster fitting (on average 04-0.6 mag) is somewhat greater than the color excess values obtained from the $\mathrm{H} \alpha / \mathrm{H} \beta$ and $\mathrm{H} \alpha / \mathrm{Pa} \alpha$ ratios presented in Sect. 4 (on average $0.4 \mathrm{mag}$ ). However, the hydrogen emission and the star clusters do not come from the same physical locations. The extinction that is found for the star clusters is confined to the star cluster region, and can thus be higher than the more global extinction map derived from the hydrogen lines.

\subsection{Stellar populations from SAURON IFU}

The star clusters discussed above (Sect. 6.1) have ages of several hundred Myr and are visible thanks to their concentrated light. How do the star clusters relate to the underlying diffuse stellar populations in the ring? Does the ring have a different star formation history from the disk, or does the disk contain similar stellar populations? With the SAURON IFU dataset, these questions can be addressed.

Similar to the work by Stoklasová et al. (2009), we fit a combination of "young" and "old" single stellar population (SSP) spectra to each Vonoroi cell ${ }^{5}$ of the SAURON mosaic using pPXF

\footnotetext{
5 At the ring positions, the size of a Vonoroi cell is equal to the origin spaxel.
} 
and GANDALF with similar settings to those in Sect. 2.3. The goal is to split the light at each position into its "young" and "old" stellar population fractions. The assumption here is that the stellar content observed in each cell can be approximated by a finite, small combination of SSPs. Thanks to the continuous evolution of stars over time, this assumption can usually be made, as long as the combination is chosen to remain sensitive to the full possible age range $(0-13$ Gyr). SSP spectra spanning 0-13 Gyr were extracted from the MILES database ${ }^{6}$ (Sánchez-Blázquez et al. 2006; Falcón-Barroso et al. 2011) with a spectral resolution of $\sigma=60 \mathrm{~km} \mathrm{~s}^{-1}$ and spectral sampling of $1.15 \AA /$ pixel. To avoid an age-metallicity degeneracy, only solar metallicity SSP models were selected. The original fit of the SAURON data, discussed in Sect. 2.3 was more extensive, and a range of metallicities was used there. It showed that solar metallicity is the dominant metallicity and thus a valid simplification here. Then, using mock observations generated from the MILES SSP spectra, together with added noise, the set of SSP spectra that still reasonably span the full possible age range could be restricted to four SSP spectra. Their age identifiers in the MILES database are 70.8 Myr, 158.5 Myr, 398.1 Myr, and 3.2 Gyr. From here on, they are designated "young", "intermediate I", "intermediate II", and "old". These four spectra will obviously not give the same age refinement as the full set, but our main goal, as stated, is to separate "young" and "old" stellar light at each spaxel/Vonoroi cell. Each Vonoroi cell in the SAURON mosaic was fit with a combination of these four SSPs.

The spectral fitting routine pPXF gives a (normalized) number-weighted output of the SSPs used to fit the spectra at each position. We used a "bootstrap"-like method to obtain error estimates on the output generated by fitting the four SSPs to each spaxel/Vonoroi cell. A measure for the fitting residual was obtained from the fit of the data with a full set of SSP models. This "residual", which is approximately $3 \%$ of the flux, was used to introduce random noise of this magnitude into the spectra. The fitting was iterated 30 times, each time introducing new random noise. Again based on mock data, the uncertainty of the number weighted fractional contribution by each of the four SSPs is estimated to be within $10 \%$ after 30 iterations. We notice from the mock data that if a large "old" population is present, the fitting underestimates the "young" fraction by several percent. The opposite is not true. The results therefore remain sensitive to older populations also in regions dominated by light from "young" stellar populations. Furthermore, the intermediate fractions are slightly underpredicted in all cases to the advantage of the "young" and "old" fractions. The fitting routine attempts to minimize the number of SSPs necessary where possible. The normalized number-weighted output is transformed into (again normalized) luminosity fractions, with the known luminosity of each SSP spectrum. The luminosity fractions can also be transformed into normalized stellar mass fractions, by use of the M/L ratio tabulated for each SSP spectrum. Finally, we multiply the normalized mass fractions with the stellar intensity at each cell (Fig. 3, top-left panel) to obtain a common baseline for all spaxels. This can be interpreted as an "absolute" mass as a function of spatial position for each age bin. The resulting maps for the four age bins are shown in Fig. 8 .

As can be seen from Fig. 8 (results have been corrected for inclination), the ring is prominent in the "young" bin, comprising about $30-40 \%$ of the mass at its galactocentric distance. The contact-points between the large-scale bar induced gas lanes and the ring stand out at the north and south positions in the ring.

\footnotetext{
6 http://miles.iac.es
}

The mass of "young" stars is highest at those positions. In the "old" bin the opposite picture is observed: almost no "old" stellar population is clearly associated with the ring, the "old" stars are distributed in a typical bulge-like profile. Significant mass in "intermediate" age stellar populations is found at the inner edge of the ring.

Figure 8 shows that the ring is not circular, but slightly ellipsoidal, when corrected for inclination. Ellipticity is very common for the entire population of circumnuclear rings (e.g. Buta \& Crocker 1993; Comerón et al. 2010).

\section{Discussion}

\subsection{Star formation signatures in the ring}

By combining the results from the star cluster analysis and the underlying stellar "field" population through the IFU fitting, the stellar content in and around the circumnuclear ring has been fully constrainted. What does this say about the manner in which star formation proceeds in this circumnuclear ring? Is there an azimuthal age gradient, as in the "pearls-on-a-string" scenario proposed by Böker et al. (2008), or has star formation resulted in a homogeneous ("popcorn") stellar age distribution (Elmegreen 1994)?

The recent star formation episodes are concentrated in the ring region (see Fig. 8). This is in accordance with our opening statement that the circumnuclear ring is the gas reservoir for the circumnuclear region. The "old" stellar bin in Fig. 8 mainly shows a bulge-like profile, with no additional mass at the ring radius itself. The IFU fitting shows a significant "young" mass fraction in the ring itself, between $30 \%$ and $40 \%$. The average age of the star clusters in the ring is young/intermediate; 200-300 Myr. This discrepancy in age (range) between the two methods is partly due to the IFU method, which slightly underpredicts the intermediate stellar fraction in favor of the "young" fraction. However, the IFU fitting also covers the full ring and is thus equally sensitive to star formation that did not lead to the formation of $\geq 10^{4} M_{\odot}$ star clusters (the lightest clusters in our sample). The ring could be currently in a state where star formation leads to $\leq 10^{4} M_{\odot}$ clusters. Possibly, the current gas inflow rate is lower than $\geq 100 \mathrm{Myr}$ ago when the detected star clusters were formed.

Both the star cluster and IFU results show that the distribution of the "young" stars is not uniform throughout the ring. The relation between the contact points and star formation is the main observable for the "pearls-on-a-string" scenario for star formation. The locations with the highest mass in "young" stars are close to the gas inflow points, the contact points, which is a point in favor of the "pearls-on-a-string" scenario.

In Fig. 9 we further plot the intensity distributions of the molecular gas tracers $\mathrm{CO}(2-1)$ and $\mathrm{HCN}(1-0)$ as a function of azimuthal angle in the ring. The azimuthal distribution of the "young" stellar mass fraction (in percentage of the total mass at each position, based on the IFU results) is also plotted there. The highest concentrations of molecular gas are close to the contact points in the north $\left(\phi \sim 180^{\circ}\right)$ and to a lesser extent in the south $\left(\phi \sim 350^{\circ}\right)$. The variations are especially pronounced in the HCN distribution. The fraction of the total mass in "young" stars by comparison shows much less variation.

The highest concentrations of "young" stars are found in the NW and SE segments of this figure. The wave pattern of the large-scale stellar bar is slower $\left(28.9 \mathrm{~km} \mathrm{~s}^{-1} / \mathrm{kpc}\right.$ pattern speed) than the stellar and gas velocity $\left(\sim 150 \mathrm{~km} \mathrm{~s}^{-1}\right)$ at the circumnuclear ring radius $(580 \mathrm{pc})$. This means that the overdensity 


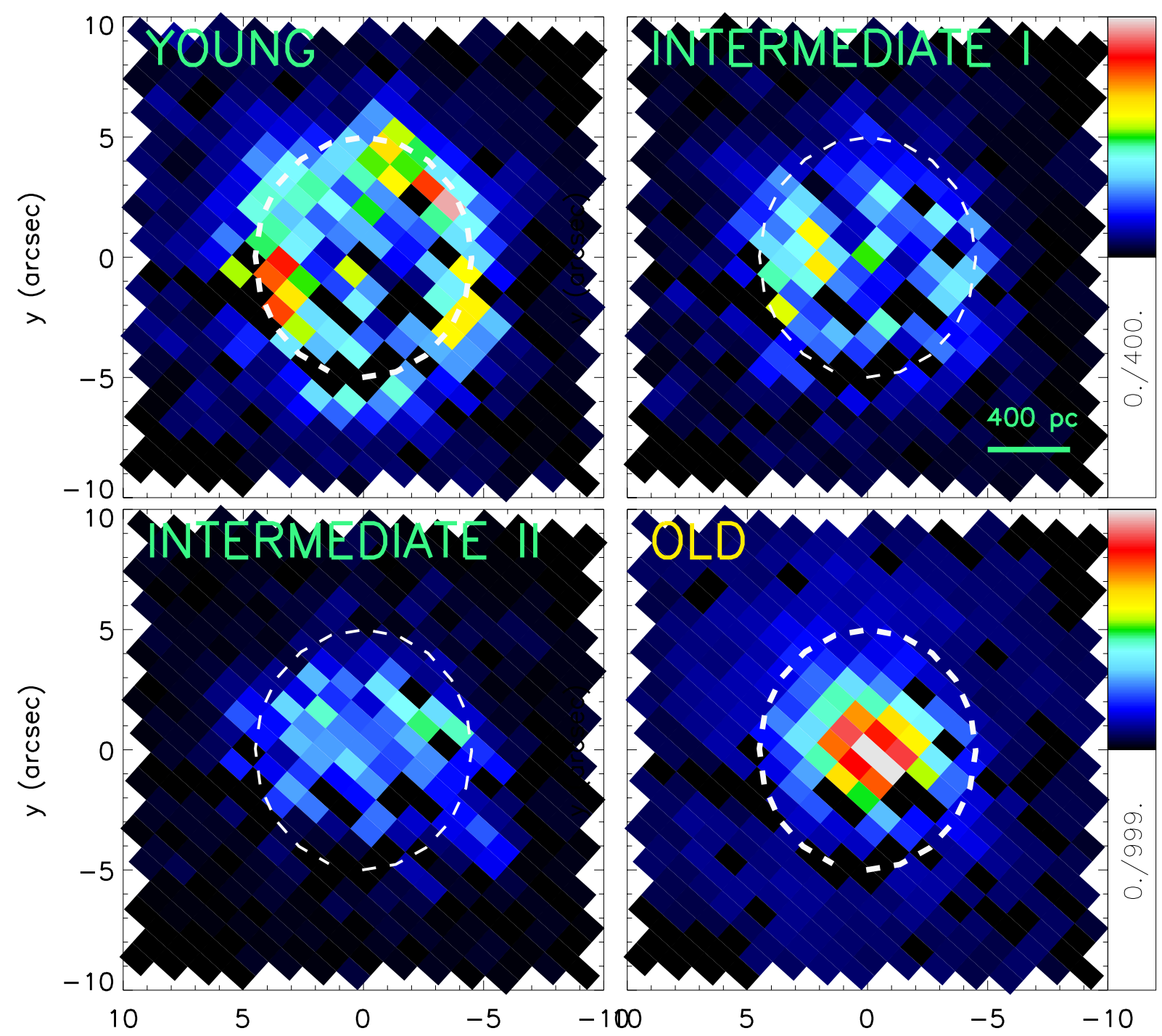

Fig. 8. Stellar "mass" maps in [AU] of mass by age bin obtained by fitting the SAURON IFU dataset with a combination of 4 SSP spectra. The maps are corrected for inclination and are oriented north up, east left. The approximate location $\left(a \sim 5^{\prime \prime}, b \sim 4.5^{\prime \prime}\right)$ of the ring is indicated by the dashed ellipse. The "mass" uncertainty is within $10 \%$.

points will move counter-clockwise in the inertial frame of the galaxy (galaxy rotation is clockwise). If stars form primarily at or near the overdensity points, the stars and gas will move faster and leave the overdensity region. The fraction of "young" stellar mass must thus be higher in front of the overdensity region (in Fig. 9 "in front of" equals at lower azimuthal angle). The offset between the highest fractions of "young" stars and the peak in $\mathrm{HCN}$ emission in the north is $\sim 20^{\circ}$. Approximately $1.5 \mathrm{Myr}$ would be needed to travel $20^{\circ}$ at the ring radius.

A half (two-fold symmetry) orbit of the bar wave pattern in the ring takes approximately $100 \mathrm{Myr}$, and stars that have formed at those locations will make five full orbits around the ring before the pattern rotation is complete. Any age signature will therefore be severely smeared within that $100 \mathrm{Myr}$ pattern time and erase the "pearls-on-a-string" scenario in favor of the "popcorn" one. The small indications of young fractions we find are thus all we can detect.

\subsection{Age of the circumnuclear ring}

The stellar age content of the ring can assist in determining of the age of the ring as a dynamical structure. Taking the stellar ages, gas inflow rate, and dynamical times together allows both a minimum and a maximum age to be constrained.

The lack of extra mass in the ring in the old stellar bin in Fig. 8 suggests that the ring cannot be older than $\sim 3$ Gyr. The average age of the star clusters is 200-300 Myr with some older (up to 1 Gyr). Van der Laan et al. (2011) have already inferred a minimum age of the large-scale stellar bar and thus the ring of $1.1 \mathrm{Gyr}$, based on mass inflow and total molecular gas mass (after helium correction).

The inflow rate determined in Van der Laan et al. (2011) was $2.0 M_{\odot} / y r$. The total mass in the star clusters we found is $\sim 6 \times$ $10^{7} M_{\odot}$. A star (cluster) formation rate history, based on the star cluster results, is given in Fig. 10. The highest SFR was reached $800 \mathrm{Myr}$ ago, after which the ring was quiescent for $300 \mathrm{Myr}$. Star formation has again started in the past 400 Myr. Over $1 \mathrm{Gyr}$ (the oldest measured star cluster age), the average star (cluster) formation rate was $\sim 0.06 M_{\odot} / y$. A star formation efficiency of a few percent is entirely plausible. That implies that the current inflow rate is a good estimate of the average inflow rate over the past $1 \mathrm{Gyr}$, even though the star formation rate was variable, and that the previously inferred minimum age of $1.1 \mathrm{Gyr}$, based on that inflow rate, is still plausible. If the star formation efficiency 
T. P. R. van der Laan et al.: Star formation in the starburst ring of NGC 6951

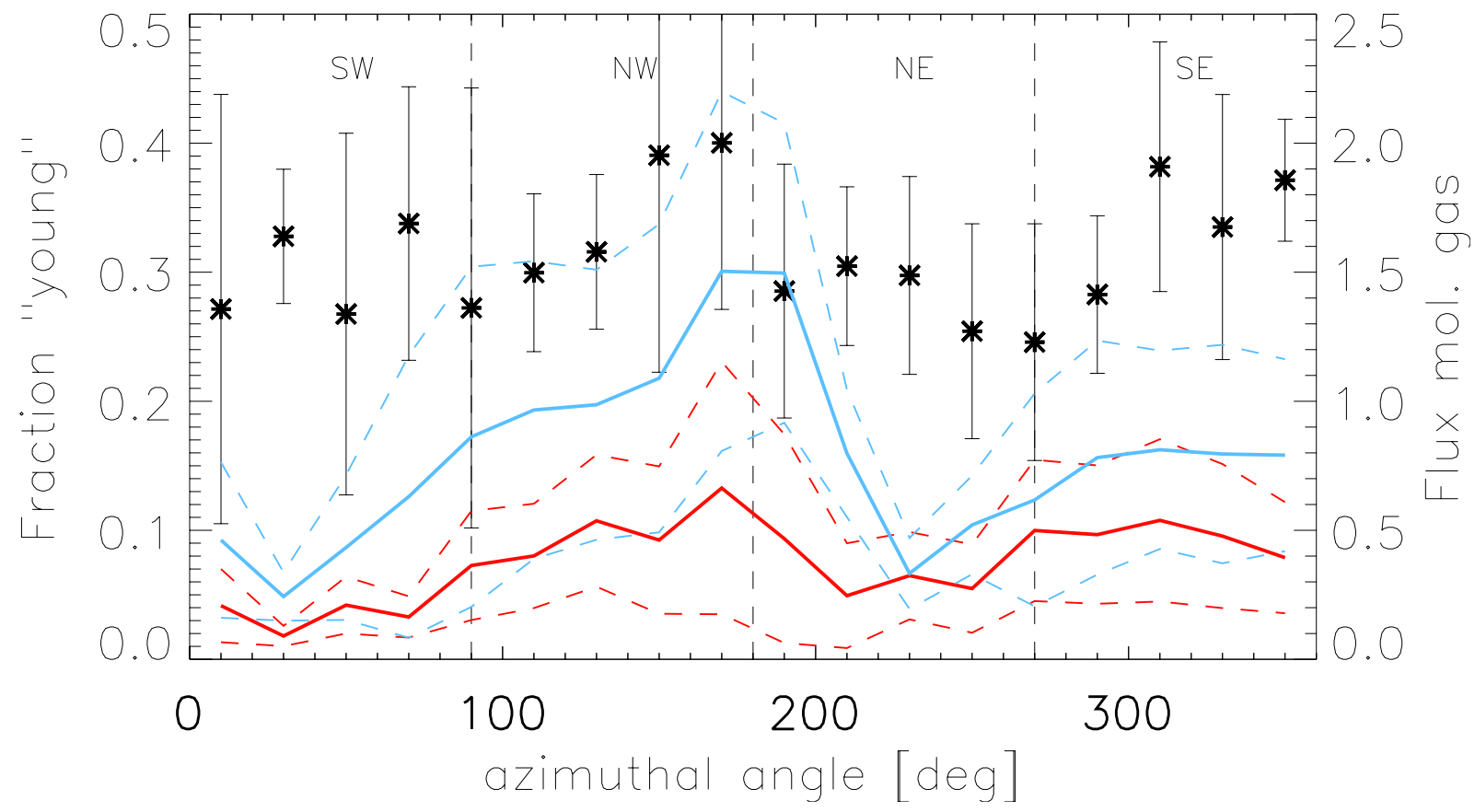

Fig. 9. Azimuthal mass fraction of young stars (asterisk), combined with the azimuthal intensity distribution of $\mathrm{CO}(2-1)$ (red line, dashed \pm 1 standard deviation) and $\mathrm{HCN}(1-0)$ emission (blue line, dashed \pm 1 standard deviation). The angle 0 corresponds to the south, angle increases counter-clockwise.

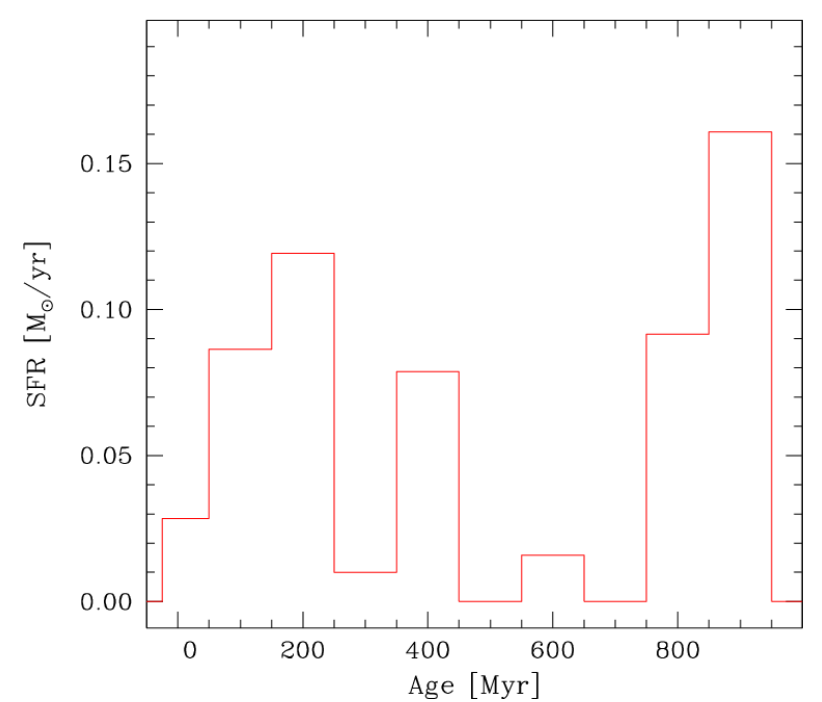

Fig. 10. Star formation rate history in the circumnuclear ring. The masses of the star clusters are grouped in $100 \mathrm{Myr}$ age bins and divided by the width of the age bin. No correction has been applied for cluster disruption, which would increase the SFR at older ages.

is higher, a lower inflow rate would suffice and the minimum age would be higher. The fraction of angular momentum lost by gas per orbit, $\mathrm{d} L / L$, is $0.2-0.4$ (Haan et al. 2009), which means gas needs at least three to five orbits to settle on the ring, which equals $200 \mathrm{Myr}$ (orbit times are larger at larger radii).

The oldest likely age for the ring is $3 \mathrm{Gyr}$, given the lack of a significant number of "old" age stars from the analysis in Sect. 6.2. A ring of 3 Gyr would imply that the ring was quietly accumulating gas for up to 2 Gyr before star formation in the ring commenced. Assuming a constant mass inflow of $2.0 M_{\odot} / \mathrm{yr}$ and a ring of $600 \mathrm{pc}\left(5^{\prime \prime}\right)$ radius and $100 \mathrm{pc}\left(1^{\prime \prime}\right)$ width, that would imply an absurdly high molecular gas surface density of $11000 M_{\odot} / \mathrm{pc}^{2}$ at the onset of star formation. That is much higher than the average $100 M_{\odot} / \mathrm{pc}^{2}$ value of most giant molecular clouds (e.g. Bigiel et al. 2008). To reach an average surface density of $100 M_{\odot} / \mathrm{pc}^{2}$ would thus only take $19 \mathrm{Myr}$.

The current age of the circumnuclear ring is therefore most likely between 1.0 Gyr and 1.5 Gyr.

\section{Conclusions}

In this work the properties of the stellar populations of the circumnuclear ring in NGC 6951 were investigated. The goals were to estimate the age of the circumnuclear ring itself, to determine the age of the stellar population(s) present in the circumnuclear ring, and to determine whether the "popcorn" or "pearls-on-astring" scenario holds true for the nature of star formation in the circumnuclear ring. The main results can be summarized as follows.

The ring is most likely between $1.0 \mathrm{Gyr}$ and $1.5 \mathrm{Gyr}$ old. It has a stellar population distinct from the rest of the circumnuclear region that was formed over that time frame. The maximum possible age for the ring is 3 Gyr. However, it is very unlikely that the ring has been in place for that long without forming stars for its first $1.5 \mathrm{Gyr}$.

Analysis of the general stellar content of the ring, by means of the SAURON mosaic, shows that $30-40 \%$ of the stellar mass in the ring is from "young" ( $<100 \mathrm{Myr})$ stars. The largest fractions are found near the contact points that link the ring with the gas lanes induced by the large-scale stellar bar.

The majority of the star clusters in the circumnuclear ring have intermediate ages. The youngest star clusters ( 100-200 Myr), when the results are averaged over $45^{\circ}$ intervals, are in the north and south, close to the contact points were gas flows onto the ring. Such a connection is indicative of the "pearls-on-a-string" scenario for star formation in circumnuclear rings. However, the dynamical time of the ring is short: it takes only 24 Myr for a star cluster to complete one rotation of the 
ring, while the age gradient can only be renewed every $100 \mathrm{Myr}$. Therefore we do not find definite evidence of the "pearls-on-astring" scenario in the circumnuclear ring of NGC 6951.

Acknowledgements. The authors thank the referee, Jesús Falcón-Barroso, for helpful comments that improved this paper. The authors also thank M. Krips for sending a version of her HCN map. We acknowledge the ING for supporting SAURON and executing the OASIS observations in service mode. Some of the data presented in this paper were obtained from the Multimission Archive at the Space Telescope Science Institute (MAST). STScI is operated by the Association of Universities for Research in Astronomy, Inc., under NASA contract NAS526555. Support for MAST for non-HST data is provided by the NASA Office of Space Science via grant NNX09AF08G and by other grants and contracts. T.vd.L. wishes to thank A. Adamo for valuable help and discussion of $\chi^{2}$ fitting. TvdL acknowledges DFG-funding (grant SCHI 536/2-3). R.Mc.D. is supported by the Gemini Observatory, which is operated by the Association of Universities for Research in Astronomy, Inc., on behalf of the international Gemini partnership of Argentina, Australia, Brazil, Canada, Chile, UK, and the USA.

\section{References}

Aguerri, J. A. L., Beckman, J. E., \& Prieto, M. 1998, AJ, 116, 2136 Athanassoula, E. 1992a, MNRAS, 259, 328

Athanassoula, E. 1992b, MNRAS, 259, 345

Bacon, R., Copin, Y., Monnet, G., et al. 2001, MNRAS, 326, 23

Barth, A. J., Ho, L. C., Filippenko, A. V., \& Sargent, W. L. 1995, AJ, 110, 1009

Bertin, E., \& Arnouts, S. 1996, A\&AS, 117, 393

Bigiel, F., Leroy, A., Walter, F., et al. 2008, AJ, 136, 2846

Böker, T., Falcón-Barroso, J., Schinnerer, E., Knapen, J. H., \& Ryder, S. 2008, AJ, 135, 479

Buta, R., \& Crocker, D. A. 1993, AJ, 105, 1344

Calzetti, D., Armus, L., Bohlin, R. C., et al. 2000, ApJ, 533, 682

Cappellari, M., \& Emsellem, E. 2004, PASP, 116, 138

Comerón, S., Knapen, J. H., Beckman, J. E., et al. 2010, MNRAS, 402, 2462

de Vaucouleurs, G., de Vaucouleurs, A., Corwin, Jr., H. G., et al. 1991, Third Reference Catalogue of Bright Galaxies

Dumas, G., Mundell, C. G., Emsellem, E., \& Nagar, N. M. 2007, MNRAS, 379, 1249

Elmegreen, B. G. 1994, ApJ, 425, L73

Emsellem, E., Cappellari, M., Peletier, R. F., et al. 2004, MNRAS, 352, 721
Englmaier, P., \& Shlosman, I. 2000, ApJ, 528, 677

Falcón-Barroso, J., Sánchez-Blázquez, P., Vazdekis, A., et al. 2011, A\&A, 532, A95

García-Burillo, S., Combes, F., Schinnerer, E., Boone, F., \& Hunt, L. K. 2005, A\&A, 441, 1011

Haan, S., Schinnerer, E., Emsellem, E., et al. 2009, ApJ, 692, 1623

Hsieh, P.-Y., Matsushita, S., Liu, G., et al. 2011, ApJ, 736, 129

Hummer, D. G., \& Storey, P. J. 1987, MNRAS, 224, 801

Hunt, L. K., \& Malkan, M. A. 2004, ApJ, 616, 707

Kim, W.-T., Seo, W.-Y., Stone, J. M., Yoon, D., \& Teuben, P. J. 2012, ApJ, 747, 60

Kohno, K., Kawabe, R., \& Vila-Vilaró, B. 1999, ApJ, 511, 157

Krips, M., Neri, R., García-Burillo, S., et al. 2007, A\&A, 468, L63

Leitherer, C., Schaerer, D., Goldader, J. D., et al. 1999, ApJS, 123, 3

Leitherer, C., Ortiz Otálvaro, P. A., Bresolin, F., et al. 2010, ApJS, 189, 309

Maciejewski, W. 2004, MNRAS, 354, 892

Mazzuca, L. M., Knapen, J. H., Veilleux, S., \& Regan, M. W. 2008, ApJS, 174, 337

McDermid, R., Bacon, R., Adam, G., Benn, C., \& Cappellari, M. 2004, in SPIE Conf. Ser. 5492, eds. A. F. M. Moorwood, \& M. Iye, 822

Mulchaey, J. S., \& Regan, M. W. 1997, ApJ, 482, L135

Osterbrock, D. E. 1989, Astrophysics of gaseous nebulae and active galactic nuclei (Mill Valley, CA: University Science Books)

Pérez, E., Márquez, I., Marrero, I., et al. 2000, A\&A, 353, 893

Regan, M. W., \& Teuben, P. 2003, ApJ, 582, 723

Saikia, D. J., Phookun, B., Pedlar, A., \& Kohno, K. 2002, A\&A, 383, 98

Sánchez-Blázquez, P., Peletier, R. F., Jiménez-Vicente, J., et al. 2006, MNRAS, 371,703

Sarzi, M., Falcón-Barroso, J., Davies, R. L., et al. 2006, MNRAS, 366, 1151

Stetson, P. B. 1987, PASP, 99, 191

Stetson, P. B. 1992, in Astronomical Data Analysis Software and Systems I, eds. D. M. Worrall, C. Biemesderfer, \& J. Barnes, ASP Conf. Ser., 25, 297

Stetson, P. B., Davis, L. E., \& Crabtree, D. R. 1990, in CCDs in astronomy, ed. G. H. Jacoby, ASP Conf. Ser., 8, 289

Stoklasová, I., Ferruit, P., Emsellem, E., et al. 2009, A\&A, 500, 1287

Storchi-Bergmann, T., Dors, Jr., O. L., Riffel, R. A., et al. 2007, ApJ, 670, 959

Tremaine, S., \& Weinberg, M. D. 1984, ApJ, 282, L5

Tully, R. B. 1988, Nearby galaxies catalog (Cambridge: Cambridge University Press)

Van der Laan, T. P. R., Schinnerer, E., Boone, F., et al. 2011, A\&A, 529, A45

Vázquez, G. A., \& Leitherer, C. 2005, ApJ, 621, 695 
T. P. R. van der Laan et al.: Star formation in the starburst ring of NGC 6951

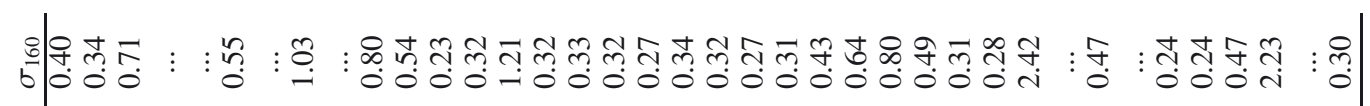

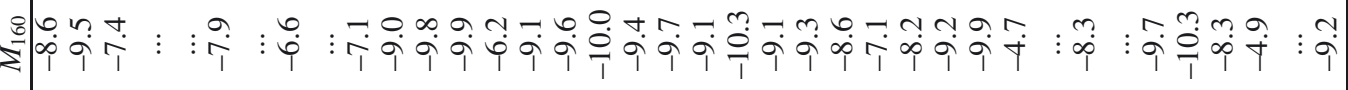

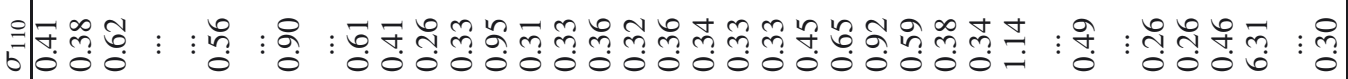

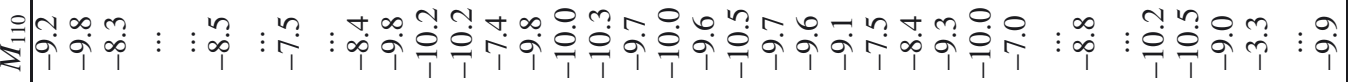

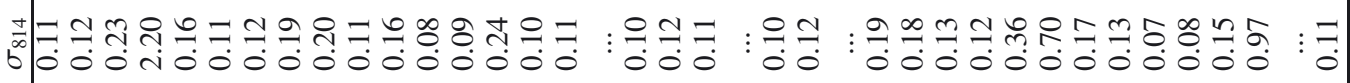

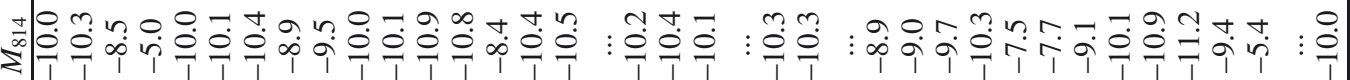

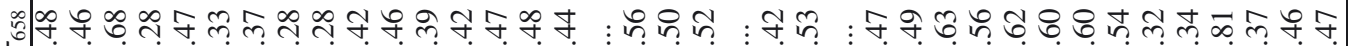

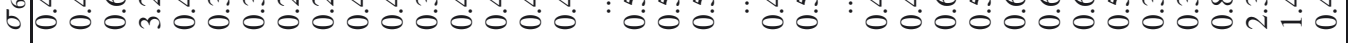
$\mathfrak{z}_{0}$

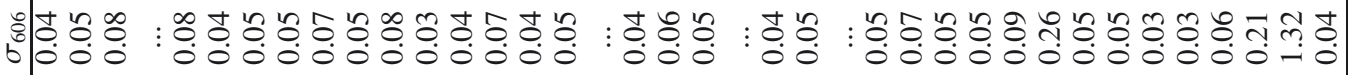

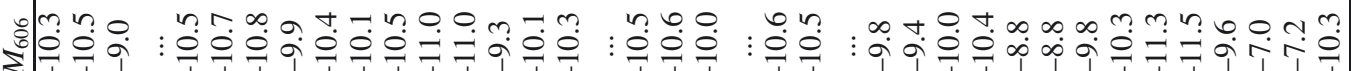

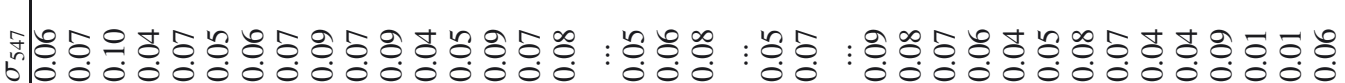

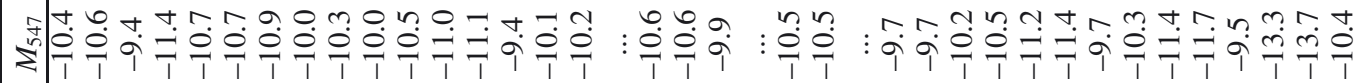

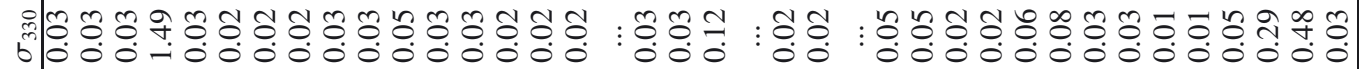

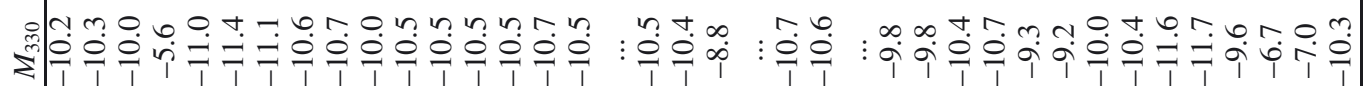
Dtta-thogagan-

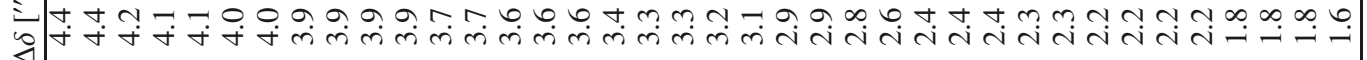

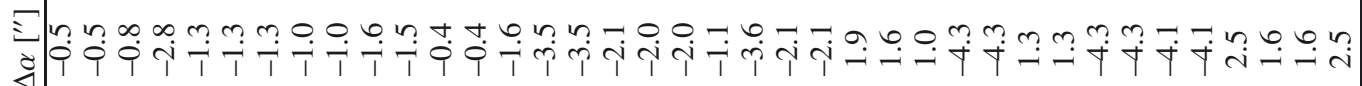

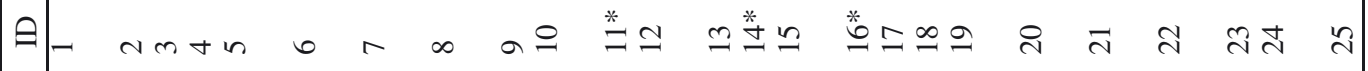


A\&A 551, A81 (2013)

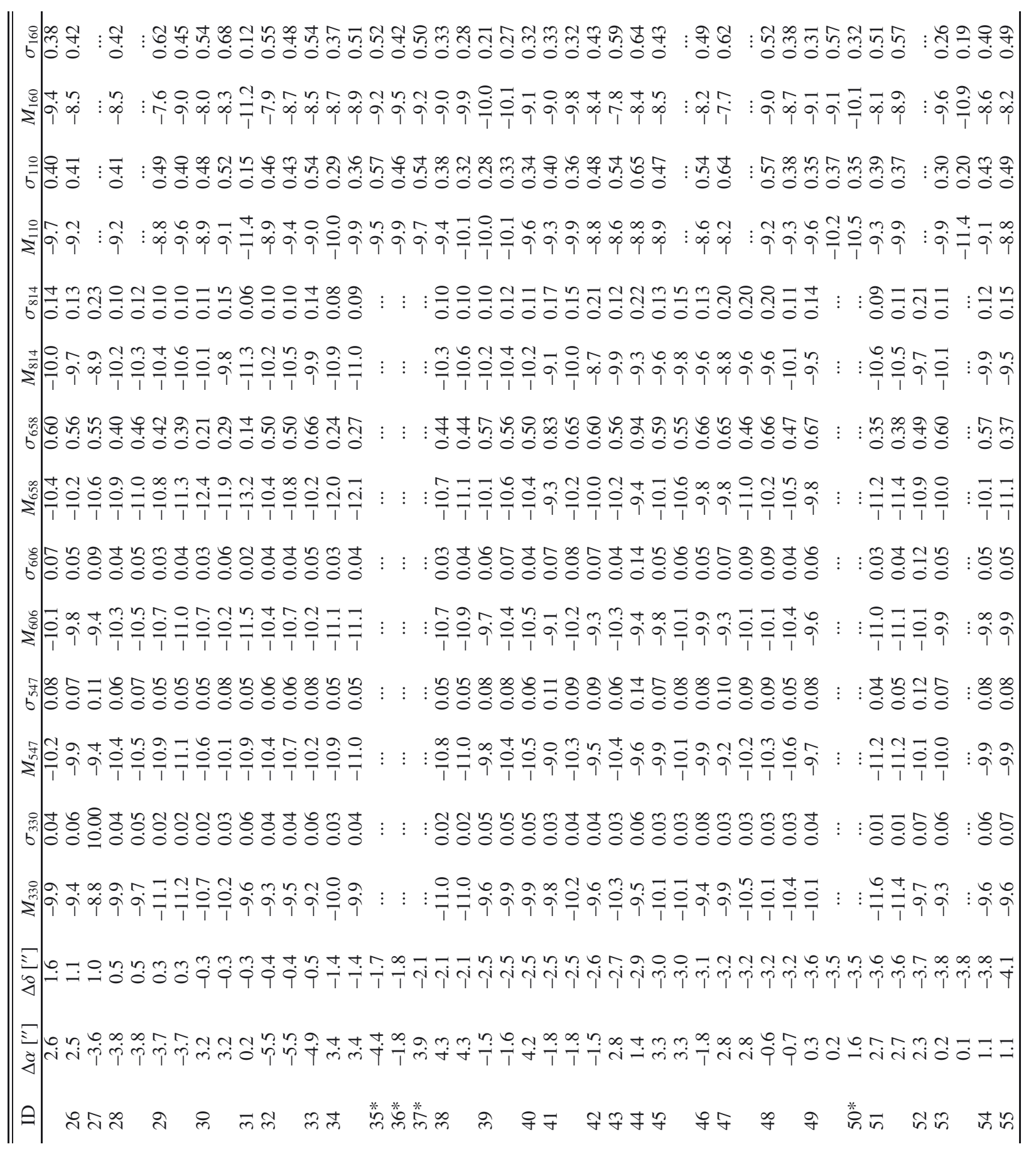


T. P. R. van der Laan et al.: Star formation in the starburst ring of NGC 6951

Table 5. Star cluster ages, extinctions, and masses.

\begin{tabular}{|c|c|c|c|c|}
\hline ID & Age [Myr] & $E(B-V)$ & $\log \left(\right.$ Mass $\left./ M_{\odot}\right)$ & $\chi^{2} /$ d.f. \\
\hline 1 & 265 & 0.44 & 5.9 & 0.56 \\
\hline 2 & 36 & 0.02 & 4.6 & 1.13 \\
\hline 3 & 964 & 0.00 & 6.4 & 5.01 \\
\hline 4 & 88 & 0.38 & 5.6 & 0.60 \\
\hline 5 & 78 & 0.35 & 5.6 & 3.90 \\
\hline 6 & 60 & 1.15 & 5.5 & 14.08 \\
\hline 7 & 184 & 0.80 & 6.0 & 2.50 \\
\hline 8 & 440 & 0.62 & 6.4 & 1.25 \\
\hline 9 & 6 & 0.31 & 4.0 & 2.87 \\
\hline 10 & 75 & 1.14 & 5.8 & 3.71 \\
\hline 12 & 220 & 0.72 & 6.1 & 1.11 \\
\hline 13 & 998 & 0.93 & 6.4 & 1.11 \\
\hline 15 & 130 & 0.90 & 6.0 & 0.72 \\
\hline 17 & 196 & 0.50 & 5.6 & 4.20 \\
\hline 18 & 130 & 0.23 & 5.2 & 2.02 \\
\hline 19 & 122 & 0.40 & 5.6 & 1.54 \\
\hline 20 & 864 & 0.00 & 6.1 & 174.29 \\
\hline 21 & 130 & 0.56 & 5.6 & 1.65 \\
\hline 22 & 140 & 0.41 & 6.1 & 0.37 \\
\hline 23 & 148 & 0.83 & 5.6 & 0.11 \\
\hline 24 & 265 & 0.00 & 6.8 & 340.26 \\
\hline 25 & 234 & 0.65 & 5.9 & 1.52 \\
\hline 26 & 420 & 0.43 & 5.8 & 1.24 \\
\hline 27 & 4 & 1.26 & 4.6 & 0.84 \\
\hline 28 & 700 & 0.49 & 6.2 & 2.29 \\
\hline 29 & 135 & 0.30 & 5.8 & 3.29 \\
\hline 30 & 134 & 1.00 & 6.0 & 21.18 \\
\hline 31 & 828 & 0.78 & 6.8 & 125.41 \\
\hline 32 & 998 & 0.75 & 6.5 & 6.87 \\
\hline 33 & 998 & 0.44 & 6.2 & 1.68 \\
\hline 34 & 996 & 0.90 & 6.8 & 8.48 \\
\hline 38 & 145 & 0.51 & 5.9 & 0.54 \\
\hline 39 & 420 & 1.04 & 6.4 & 4.04 \\
\hline 40 & 490 & 0.60 & 6.2 & 0.12 \\
\hline 41 & 100 & 1.04 & 5.5 & 2.45 \\
\hline 42 & 136 & 0.31 & 5.2 & 1.32 \\
\hline 43 & 216 & 0.42 & 5.8 & 1.15 \\
\hline 44 & 190 & 0.69 & 5.6 & 0.37 \\
\hline 45 & 162 & 0.50 & 5.6 & 0.49 \\
\hline 46 & 450 & 0.36 & 5.8 & 0.52 \\
\hline 47 & 64 & 0.64 & 5.3 & 0.53 \\
\hline 48 & 241 & 0.21 & 5.7 & 0.48 \\
\hline 49 & 122 & 0.43 & 5.4 & 5.49 \\
\hline 51 & 100 & 0.18 & 5.7 & 1.97 \\
\hline 52 & 336 & 0.72 & 6.0 & 0.68 \\
\hline 53 & 836 & 0.62 & 6.2 & 5.88 \\
\hline 54 & 248 & 1.04 & 6.0 & 2.38 \\
\hline 55 & 294 & 0.58 & 5.8 & 2.85 \\
\hline
\end{tabular}

Notes. Results of the $\chi^{2}$ fitting of age (Col. 2), color excess (Col. 3), and mass (Col. 4) of the observed star clusters. Only those candidates that were successfully fitted are listed. The star cluster ID in Col. 1 is the same as those in Table 4. 